\title{
A QUANTITATIVE AND TEMPORAL MAP OF PROTEOSTASIS DURING HEAT SHOCK IN SACCHAROMYCES CEREVISIAE
}

DOI:

10.1039/c7mo00050b

\section{Document Version}

Accepted author manuscript

Link to publication record in Manchester Research Explorer

Citation for published version (APA):

Jarnuczak, A., Garcia Albornoz, M., Eyers, C. E., Grant, C., \& Hubbard, S. (2018). A QUANTITATIVE AND

TEMPORAL MAP OF PROTEOSTASIS DURING HEAT SHOCK IN SACCHAROMYCES CEREVISIAE. Molecular Omics, 14, 37-52. https://doi.org/10.1039/c7mo00050b

\section{Published in:}

Molecular Omics

\section{Citing this paper}

Please note that where the full-text provided on Manchester Research Explorer is the Author Accepted Manuscript or Proof version this may differ from the final Published version. If citing, it is advised that you check and use the publisher's definitive version.

\section{General rights}

Copyright and moral rights for the publications made accessible in the Research Explorer are retained by the authors and/or other copyright owners and it is a condition of accessing publications that users recognise and abide by the legal requirements associated with these rights.

\section{Takedown policy}

If you believe that this document breaches copyright please refer to the University of Manchester's Takedown Procedures [http://man.ac.uk/04Y6Bo] or contact uml.scholarlycommunications@manchester.ac.uk providing relevant details, so we can investigate your claim.

\section{OPEN ACCESS}




\section{A QUANTITATIVE AND TEMPORAL MAP OF PROTEOSTASIS DURING}

2 HEAT SHOCK IN SACCHAROMYCES CEREVISIAE

3

4 Andrew F. Jarnuczak ${ }^{1,3}$, Manuel Garcia Albornoz ${ }^{1}$, Claire E. Eyers ${ }^{2}$, Christopher M. Grant ${ }^{1}$,

5 Simon J. Hubbard ${ }^{*}$

6 1. School of Biological Sciences, Faculty of Biology, Medicine and Health, University of Manchester, Manchester Academic Health Science Centre, Oxford Road, Manchester M13 9PT

2. Centre for Proteome Research, University of Liverpool, Department of Biochemistry, Institute of Integrative Biology, Liverpool, L69 7ZB, UK

3. European Bioinformatics Institute (EMBL-EBI), Wellcome Trust Genome Campus, Hinxton, Cambridge, United Kingdom

$12{ }^{*}$ Corresponding author: simon.hubbard@manchester.ac.uk

14 Competing interests: There are no competing interests from any of the authors.

15

16 Keywords: time course, quantitative proteomics, environmental stress response, label free

17 quantification, HSP70 chaperone 


\section{ABSTRACT}

21 Temperature fluctuation is a common environmental stress that elicits a molecular response in order to maintain intracellular protein levels. Here, for the first time, we report a comprehensive temporal and quantitative study of the proteome during a 240-minute heat stress, using label-free mass spectrometry. We report temporal expression changes of the hallmark heat stress proteins, including many molecular chaperones, tightly coupled to their protein clients. A notable lag of 30 to 120 minutes was evident between transcriptome and proteome levels for differentially expressed genes. This targeted molecular response buffers the global proteome; fewer than $15 \%$ of proteins display significant abundance change. Additionally, a parallel study in a Hsp70 chaperone mutant (ssb1 $1 \Delta$ ) demonstrated a significantly attenuated response, at odds with the modest phenotypic effects that are observed on growth rate. We cast the global changes in temporal protein expression into protein interaction and functional networks, to afford a unique, time-resolved and quantitative description of the heat shock response in an important model organism.

\section{Table of Contents}

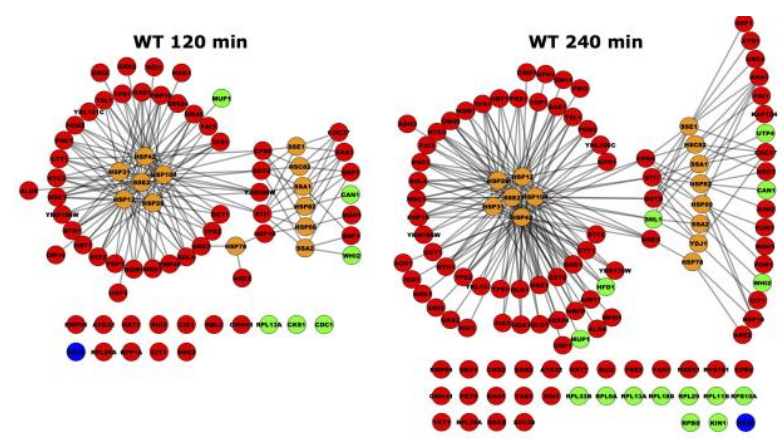

Temporal changes in the yeast proteome under heat stress are mapped and integrated to protein networks to reveal cognate groups of chaperones (orange and blue circles) acting on coherent groups of substrate proteins (red and green). 


\section{Introduction}

44 All organisms are potentially exposed to adverse environmental conditions at some point during their development. Much effort has therefore been devoted to elucidating mechanisms of stress response in both unicellular and multicellular eukaryotes ${ }^{1-4}$. The budding yeast $S$. cerevisiae has provided an important model organism contributing to our understanding of environmental stress responses at a molecular level for many decades ${ }^{5-}$ 9. Among different types of stresses, heat stress is generally very well understood ${ }^{10-12}$. Caused by sudden temperature shifts of as little as a few degrees, it can have many damaging effects and results in pronounced physiological and metabolic changes ${ }^{10,11}$. For example: increased protein unfolding and aggregation, destabilisation of cell wall or membranes, metabolic reprogramming, or cell cycle arrest ${ }^{10,11}$. In response, a widespread reorganisation of gene expression has been observed. In cases of general cellular stress (proteotoxic, osmotic, starvation, etc.) it is termed the environmental stress response $(E S R)^{8,9,13}$ and in the specific case of heat stress, it is simply called the heat shock response $(\mathrm{HSR})^{10,11}$.

The key protein players in HSR are transcription factors, Hsf1p and the partially redundant Msn2p/Msn4p pair, in addition to various downstream heat-shock effector proteins which mainly act as molecular chaperones. Hsf1 binds to heat shock elements (HSE) in the promoter region of its target genes and activates their transcription ${ }^{12,14,15}$. Hsf1 targets include many chaperones, genes of the protein transport and degradation machinery, cell signalling or transcription ${ }^{16,17}$. Msn2/4 on the other hand binds to a stress responsive element (STRE) promoter motif and is also a regulator of multiple stresses ${ }^{18}$. As noted, the best known heat-induced proteins are molecular chaperones, initially discovered and characterised as heat shock proteins (Hsps). Chaperones are necessary for protein biogenesis in normal conditions by facilitating and/or assisting de novo folding or protein translocation, and in stress conditions (as well as in normal conditions) they prevent aggregation and assist in the refolding of misfolded proteins ${ }^{12}$. Hsp70s (for example, the constitutive Ssa1p/Ssa2p and stress-inducible Ssa3p/Ssa4p) and Hsp90 chaperones (Hsp82p redundant in function with its paralogue Hsc82p) with its cofactors, e.g. Sti1p, are all well documented to be induced in heat stress ${ }^{10,13}$. Incidentally, it has been proposed that Hsp70 and Hsp90 chaperones are also involved in heat stress sensing by being titrated away from Hsf1p upon accumulation of misfolded proteins ${ }^{10}$. Other proteins are also known to be upregulated in heat stress, including the disaggregase Hsp104p, 
antiaggregases Hsp26p and Hsp42p, and membrane stabilising Hsp12p ${ }^{10}$. In addition to induction of heat shock genes, some gene expression is also transcriptionally repressed. These include growth-related and protein biosynthetic genes like ribosomal components and metabolic enzymes ${ }^{9}$.

Interestingly the mRNAs encoding two ribosomally-associated Hsp70 family members, Ssb1/2, were also reported to be rapidly reduced in abundance upon shift from $23^{\circ} \mathrm{C}$ to $37^{\circ} \mathrm{C}^{19}$. Ssb1p and Ssb2p are chaperones associated with actively translating ribosomes and, together with Ssz1p and co-chaperone J-domain partner Zuo1p, form the ribosomeassociated complex (RAC). Ssb1/2p bind and assist the folding of newly synthesised polypeptide chains, possibly by assisting its transport through the ribosome tunnel or by protecting it from aggregating until other chaperones actively take over the folding ${ }^{20-22}$. Ssb1/2p have also been found to be necessary to maintain translational fidelity in yeast ${ }^{23}$. Moreover, despite being 99\% identical, differential function of Ssb1p and Ssb2p has been proposed in the literature ${ }^{24}$. For example, only Ssb1p was able to rescue a phenotype observed in Tim18p mutant cells which are unable to live without mitochondrial DNA. Additionally, in the context of this study, the lack of Ssb1p would be expected to exacerbate the problem of thermal protein misfolding and deleteriously affect translation during heat stress. This presents Ssb1p mutant cells in particular, as an interesting target for deeper investigations of their unique heat stress response.

Evidently, HSR is a complex process that requires an integrative system-wide view in order to be fully understood. However, while HSR has been very well characterised on the global transcriptome level ${ }^{8,9,25}$, perhaps surprisingly, no large-scale temporal proteome studies have so far been reported. Importantly, although no HSR proteomics surveys with temporal resolution have been performed, two relevant studies are available: a study by Nagaraj \& Kulak et.al ${ }^{26}$, and another one by Shui \& Xiong et.al ${ }^{27}$. Nagaraj \& Kulak et.al ${ }^{26}$ used a quadrupole Orbitrap mass spectrometer and a spike-in SILAC approach to quantify protein abundance changes in a wild type laboratory S288C yeast strain grown at $24^{\circ} \mathrm{C}$ and transferred to $37^{\circ} \mathrm{C}$ for half an hour. They achieved an impressive coverage of just over 3100 proteins and found many heat shock proteins to be up-regulated and ribosome biogenesis proteins down-regulated in response to elevated temperature. Additionally, evidence of regulation of translation through down-regulation of proteins implicated in translation initiation and elongation was highlighted in this study. Interestingly, the proteins found to be induced in heat shock were often highly up-regulated; for example Ssa4p had 


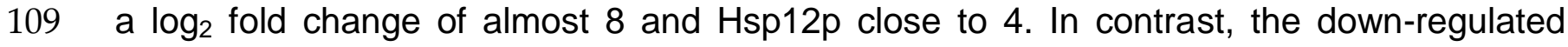
110 proteins had much smaller fold changes, often below 2 fold.

111 In a more recent study, Shui \& Xiong et.al ${ }^{27}$ used iTRAQ and 2D reversed phase 112 fractionation to investigate the proteome response to prolonged heat exposure in two 113 thermotolerant industrial strains: ScY01 and its parental strain ScY. Comparisons with the 114 Nagaraj et.al ${ }^{26}$ study supported the differentiation of the thermotolerant response (TR) 115 from the heat shock response (HSR). Central carbon metabolism and lipid metabolism 116 were decreased in thermotolerance but increased in heat shock response; for example 117 Cyb2p, Gph1p, Faa1p, Hor2p, Cct1p, Aro10p were up-regulated in HSR but down118 regulated in TR.

119 Those studies however examined only a single time point during HSR. Here we use a 120 label-free quantitative proteomics approach to determine temporal changes in the yeast 121 proteome at different times during heat stress induced by a mild temperature shift, from $12230^{\circ} \mathrm{C}$ to $37^{\circ} \mathrm{C}$; a first such study. We measured proteome-level expression for the haploid, 123 a wild type (WT) strain and an isogenic strain lacking the Ssb1p chaperone (ssb1 $\Delta$ ). The 124 resulting temporal profiles confirmed many of the HSR hallmark characteristics and were 125 in excellent agreement with the literature. They also revealed additional insights not 126 available from earlier microarray-based efforts or proteomics studies lacking temporal 127 resolution. Of particular interest is that while heat stress response on the mRNA level 128 appears to be almost immediate (minutes) and with large amplitude or distinct temporal 129 patterns, a steady and uniform response (0.5 - 1 hour) on the protein level is visible, at 130 least during the mild heat stress considered here. Additionally, we analyse the temporal 131 profile of an $s s b 1 \Delta$ mutant in the same heat stress conditions and characterise at the 132 molecular level how the deletion produces a markedly attenuated response compared to 133 the WT cells. We interpret both data sets in conjunction with known chaperone-target 134 protein interactions, to reveal a global picture of the entire temporal heat shock response, 135 which demonstrates a systems-wide view of proteostasis in action.

\section{Experimental}

\section{Yeast growth and proteomics sample preparation}

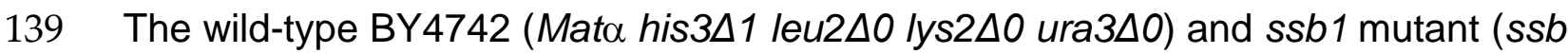


$1:: k a n M X 4)$ yeast strains used in this study were purchased from the Thermo Fisher

141 Scientific Yeast Knockout (YKO) Collection. Batch cultures were grown in Yeast Nitrogen 142 Base medium (supplemented with $10 \mathrm{~g} / \mathrm{L}$ of glucose, $10 \mathrm{ml} / \mathrm{L} \mathrm{Arg}, 20 \mathrm{ml} / \mathrm{L} \mathrm{Leu}, 10 \mathrm{ml} / \mathrm{L}$ 143 Ura, $3 \mathrm{ml} / \mathrm{L}$ His and $10 \mathrm{ml} / \mathrm{L}$ Lys) in biological quadruplicates. For the heat stress 144 experiment, WT and ssb $1 \Delta$ cells were grown at $30^{\circ} \mathrm{C}$ in glass conical flasks until they 145 reached mid-exponential growth phase $\left(\mathrm{OD}_{600} \sim 1.9\right)$ and were then moved to a large 146 rotating water bath pre-heated to $37^{\circ} \mathrm{C}$. In order to ensure the temperature equilibration 147 inside the flasks was rapid, the water level in the bath was maintained above the culture 148 level and the bath lid was closed. A $5 \mathrm{ml}$ sample of unstressed cells was removed at time $1490 \mathrm{~min}$ (T0) and then at $10 \mathrm{~min}$ (T10), $30 \mathrm{~min}$ (T30), $60 \mathrm{~min}$ (T60), $120 \mathrm{~min}$ (T120) and 240 $150 \mathrm{~min}(\mathrm{~T} 240)$ after transfer to $37^{\circ} \mathrm{C}$. For each time point four biological replicates were 151 collected and cells were counted using a Cellometer cell counter (Cellometer AUTOM10 152 by Nexcelom. http://www.nexcelom.com). Cell pellets were collected by centrifugation at $1534000 \mathrm{rpm}$, for 10 minutes at $4^{\circ} \mathrm{C}$ and stored at $-80^{\circ} \mathrm{C}$ until use.

154 Cell lysis was performed according to an earlier described protocol $^{28}$ with minor 155 modifications. Briefly, the cell pellet was re-suspended in ice-cold $50 \mathrm{mM} \mathrm{NH}_{4} \mathrm{HCO}_{3}$ with 156 added ROCHE Complete mini protease and phosphatase inhibitor cocktails (Roche 157 Diagnostics Ltd, West Sussex, UK), 1 tablet of each/10 ml of buffer, and washed twice by 158 centrifugation (at $4000 \mathrm{rpm}$ for $5 \mathrm{~min}$ at $4^{\circ} \mathrm{C}$ ) to remove any contaminants. Cell lysis was 159 achieved by automated glass bead-beating using the MINILYS® homogenizer (Precellys, 160 UK), $10 \times 1$ minute cycles at $4^{\circ} \mathrm{C}$ with 2 min break between each cycle, when lysates were 161 cooled on ice. The lysate was centrifuged (13,000 rpm, $4^{\circ} \mathrm{C}$ for 10 minutes) and protein 162 collected from supernatant. The remaining cell debris was re-suspended in lysis buffer, 163 collected by centrifugation (10 $\mathrm{min}, 16000 \mathrm{rpm}, 4^{\circ} \mathrm{C}$ ) and combined with the protein 164 supernatant.

165 For protein digestion, a volume equivalent to 25 million cells ( 100 - $150 \mu \mathrm{g}$ protein) was 166 diluted to $160 \mu \mathrm{L}$ with $25 \mathrm{mM}$ ammonium bicarbonate. Proteins were denatured with 167 RapiGest ${ }^{\mathrm{TM}}$ detergent $\left(10 \mu \mathrm{L}\right.$ of $1 \%(\mathrm{w} / \mathrm{v}), 80^{\circ} \mathrm{C}$ for 10 minutes), reduced using $60 \mathrm{mM}$ 168 dithiothreitol $\left(10 \mu \mathrm{L}, 60^{\circ} \mathrm{C}, 400 \mathrm{rpm}\right.$ shaking for 10 minutes), cooled on ice and alkylated $169180 \mathrm{mM}$ iodoacetamide (10 $\mu \mathrm{L}$, incubation at room temperature in the dark for 30 minutes). 170 Digestion was performed by addition of sequencing grade porcine trypsin (Promega, UK) 171 in a 1:50 enzyme to protein ratio (10 $\mu \mathrm{L}$ of $0.2 \mu \mathrm{g} / \mu \mathrm{L}$ trypsin), incubation for $4.5 \mathrm{~h}$ followed 172 by further trypsin addition $\left(10 \mu \mathrm{L}\right.$ of $0.2 \mu \mathrm{g} / \mu \mathrm{L}$ trypsin), and overnight incubation at $37^{\circ} \mathrm{C}$. 
173 Trifluoroacetic acid (TFA) was added to the reaction mixture (1\% (v/v) final concentration) 174 to stop the digestion and hydrolyse RapiGest ${ }^{\mathrm{TM}}$ detergent. To aid peptide solubilisation, $1752.5 \mu \mathrm{L}$ acetonitrile:water (2:1) was also added and samples were incubated at $4{ }^{\circ} \mathrm{C}$ for at 176 least 2 hours.

177 Finally, RapiGest ${ }^{\mathrm{TM}}$ precipitate and any remaining particulate in the sample was removed 178 by centrifugation ( $30 \mathrm{~min}, 13,000 \mathrm{rpm}$ at $4^{\circ} \mathrm{C}$ ). Additionally, two QC pools were prepared 179 containing an equal mix of all WT digests (WT-QC) and another one of all ssb1 digests 180 (ssb1 $\Delta-Q C)$.

181 LC-MS data acquisition

182 The digests were analysed on a nanoACQUITY UPLC ${ }^{\text {TM }}$ system (WATERS) coupled to 183 LTQ Orbitrap Velos (linear trap quadrupole with an Orbitrap mass analyser; Thermo). 184 Peptides equivalent to 306,818 cells, and 397,727 cells for the QC runs were loaded onto 185 a C-18 pre-column (Symmetry C18 Column, $100 \AA$ pore size, $5 \mu \mathrm{m}$ particle size, $180 \mu \mathrm{m} x$ $18620 \mathrm{~mm}$, Waters, Manchester, UK) and trapped for 5 minutes at a flow rate of $5 \mu \mathrm{L} / \mathrm{min}$ in 187 99.9\% solution A (water, 0.1\% (v/v) formic acid). Peptides were then resolved on an 188 analytical C18 column (nanoACQUITY UPLC ${ }^{\text {TM }}$ HSS T3 C18 $75 \mu \mathrm{m}$ x $150 \mathrm{~mm}, 1.7 \mu \mathrm{m}$ 189 particle size, Waters, Manchester, UK) with a linear gradient of solution B (acetonitrile, $1900.1 \%(\mathrm{v} / \mathrm{v})$ formic acid) from $3 \%$ to $35 \%$ over 210 minutes at a flow rate of $300 \mathrm{~nL} / \mathrm{min}$, 191 followed by a ramp to $90 \%$ solution $\mathrm{B}$, column wash and 15 minute re-equilibration. The 192 total run time was 240 minutes. A 30 min blank injection was also performed between 193 each tryptic sample to wash the columns and minimise carry-over between samples.

194 The LTQ Orbitrap was operated in data-dependent mode, with the twenty most intense 195 precursor ions (with charge state $\geq 2$ ) in the survey scan selected for collision induced 196 dissociation (CID) fragmentation. The survey scan was acquired over the range $\mathrm{m} / \mathrm{z} 350$ 1972000 at a resolution of $30,000(\mathrm{~m} / \mathrm{z} 400)$ in the Orbitrap analyser and the fragmentation was performed in the LTQ ion trap with the normalised collision energy of 35.

\section{Label-free protein quantification}

200 Mass spectrometry data was acquired in two blocks, WT and ssb1 samples separately. 201 Acquisition order within each block was randomised and a QC sample was injected at the 202 beginning, intermediately and at the end of each MS run, a total of 5 QC injections. The 203 raw instrument data was then processed using MaxQuant (MQ) software ${ }^{29}$. Protein 204 identification was performed with the built-in Andromeda search engine ${ }^{30}$. MS/MS spectra 
205 were searched against the S. cerevisiae canonical and isoform protein database (UniProt, 2066721 entries, accessed April 2015) appended with 124 common laboratory contaminants. 207 The parameters were as follows; digest reagent: trypsin, max missed cleavages: 2, max 208 protein mass: $250 \mathrm{kDa}$, fixed modifications: cysteine carboxymethylation, variable 209 modifications: protein $\mathrm{N}$-terminal acetylation and methionine oxidation and serine, 210 threonine and tyrosine (STY) phosphorylation. The mass tolerance was set to $6 \mathrm{ppm}$ for 211 MS1 and MS/MS to $0.5 \mathrm{Da}$. The initial MS1 mass calibration was performed within MQ 212 with a $20 \mathrm{ppm}$ tolerance. The false discovery rate (FDR) for accepted peptide spectrum 213 matches and protein matches was set to $1 \%$.

214 For protein quantification, 're-quantify' and 'match between runs' options were selected; 215 match time window was set to 1 min after manual inspection of average chromatographic peak widths in each dataset. Label free quantification was performed with the MaxLFQ algorithm ${ }^{31}$ within MaxQuant. Minimum ratio count was set to 1 and quantification was based on razor and unique peptides only. Razor peptides are determined based the

219 Occam's razor principle where a minimum set of proteins accounting for all identified 220 peptides is used ${ }^{32,33}$. All other $M Q$ parameters were left in default. The raw mass 221 spectrometry data was deposited to the ProteomeXchange Consortium ${ }^{34}$ with the identifier 222 PXD006262

\section{Proteomics data handling procedure}

Protein significance testing

225

In order to determine statistically significantly changing proteins with respect to time point T0 we used the MSstats package ${ }^{35}$ in combination with empirical fold change cut-offs based on technical noise in each experiment. Proteins with an adjusted $p$-value $<0.05$ and outside the fold change cut-offs were considered to change expression with respect to T0 in response to heat stress. For a detailed explanation of the procedure, see the

230 Supplementary methods description and Supplementary Figure 3.

231 The final quantification results from MSstats are available in Supplementary File 1. The 232 original, unprocessed $M Q$ output proteinGroups tables are available in Supplementary 233 File 2 (WT) and 3 (ssb1 $1 \Delta$ ). The MQ evidence.txt files are uploaded to PRIDE with the id 234 PXD006262. 
236 To identify groups of proteins with similar temporal patterns, c-means fuzzy clustering was 237 performed in $\mathrm{R}$ (http://www.R-project.org/) using the Mfuzz package ${ }^{36}$. The number of 238 clusters was determined empirically, by inspecting the results for 2-12 clusters, and 239 assessing biological validity of the resulting clusters (GO processes enrichment) as well as 240 cluster membership and the number of observations in each cluster. The $m$ parameter 241 setting was determined automatically according to Schwaemmle and Jensen ${ }^{37}$ as 242 implemented in Mfuzz package, 'mestimate' function.

243 Gene ontology enrichment analysis was performed with the online application Panther ${ }^{38,39}$, 244 directly on the Gene Ontology Consortium webpage (http://pantherdb.org/). The 245 background set consisted of all proteins identified in a given MS experiment and the target 246 set was the significantly changing proteins, or, in case of c-means fuzzy clustering, the 247 proteins within each cluster and cluster membership $>0.5$. Only terms with a q-value 248 below 0.05 were considered.

249 From the list of differentially abundant proteins, all molecular chaperones were linked to 250 their attendant protein clients based on the filtered protein interaction dataset used 251 previously $^{40,41}$. Based on this information, a bipartite network was created linking proteins 252 by their chaperone-target interactions and a protein-protein interaction network analysis 253 was created using Cytoscape ${ }^{42}$ with an organic layout. Additionally, all the differentially 254 abundant proteins were linked to their Slim GO biological process terms and a bipartite 255 network was created linking proteins by their shared GO terms from which a PPI network 256 analysis was done as before.

\section{Results}

259 Generation of MS-based temporal protein profiles

260 Our experimental strategy followed the $S$. cerevisiae protein level response to heat stress 261 induced by shift from $30^{\circ} \mathrm{C}$ to $37^{\circ} \mathrm{C}$, the classic 'heat shock' ${ }^{\prime 10}$. This temperature results in a 262 robust response but is low enough for cells to sustain growth and is physiologically 263 relevant. As expected based on previous literature ${ }^{43}$, the two strains under investigation; 264 WT (wild type, BY4742, Mat ALPHA) and chaperone mutant ssb1 (BY4742, Mat 265 ALPHA), had very similar growth rates in glucose-rich, batch cultures at normal 
266 temperature $30^{\circ} \mathrm{C}$, with estimated doubling times of $2.8 \mathrm{~h}$ and $2.9 \mathrm{~h}$, respectively 267 (Supplementary Figure 1). Upon heat stress, at $37^{\circ} \mathrm{C}$, the growth rate was slowed in both 268 strains, with estimated doubling times during the first 240 minutes increased to $3.8 \mathrm{~h}$ in WT 269 and $3.6 \mathrm{~h}$ in $s s b 1 \Delta$. The cultures were sampled in quadruplicate at $10 \mathrm{~min}$ (T10), $30 \mathrm{~min}$ 270 (T30), $60 \mathrm{~min}$ (T60), 120min (T120) and $240 \mathrm{~min}$ (T240) after transfer to $37^{\circ} \mathrm{C}$. The control 271 (mid-exponential cells, $30^{\circ} \mathrm{C}$ ) was obtained just before the shift, at time T0 (Figure 1A). 272 After cell lysis, total protein extracts were digested with trypsin and mass spectrometry 273 data was obtained in 48 single-shot LC-MS whole proteome analyses (Figure 1A), using a randomised block design ${ }^{44}$. Relative protein quantification was achieved using a label-free approach using MaxQuant ${ }^{29,31}$, achieving excellent quantification repeatability in both the 276 WT and ssb1 1 blocks of analysis (Supplementary Figure 2). Altogether, we quantified 2772141 proteins, at 1\% PSM and protein-level FDR from all WT and ssb1 $\Delta$ runs, 278 representing around $50 \%$ of the expressed yeast proteome ${ }^{45}$. After robust statistical 279 analysis (see Material and methods), 1780 proteins in WT and 1970 in ssb1 were 280 consistently quantified in each of the time points with 1609 proteins overlapping between 281 the two datasets (Figure 1B, Venn diagram). This provides good protein coverage and isoform resolution; $~ 80 \%$ proteins in both strains were identified with 3 or more unique 283 peptides across the whole dataset, with an average of 6 unique peptides per protein 284 (boxplots, Figure 1B) This supported the resolution and quantification of potentially 285 interesting protein isoforms/paralogues. For example, all of the SSA subfamily members of 286 the ubiquitously expressed HSP70 heat shock protein family could be quantified in the $287 s s b 1 \Delta$ dataset (3 out of 4; Ssa1p, Ssa2p and Ssa4p, could be quantified in the WT). 288 However, because of high sequence similarity (99\%) between Ssb isoforms, the two 289 proteins Ssb1p and Ssb2p can not be reliably distinguished by any untargeted MS-based 290 proteomic technique.

291 Temporal profiles of relative protein abundance changes upon heat stress were calculated 292 from the protein ratios at each time point with respect to time 0, represented by a heatmap 293 for each strain shown side-by-side in Figure 2. Each cell represents the model-based 294 estimate of fold change from MSstats at a given time (expressed as $\log _{2}$ of the ratio) after 295 transfer to $37^{\circ} \mathrm{C}$. The protein profiles for the WT and mutant cells were additionally 296 clustered row-wise using Euclidean distance. This overview shows the global proteomic 297 response to heat stress in the WT and mutant cells, with many proteins increasing (red) and decreasing (blue) in relative abundance post-stress; however, most show a change of 
299 less than 1.5 fold (corresponding to $\log _{2}=0.58$ ). Notably, many proteins display co300 ordinated but significantly different behaviour in the mutant cells compared to wild type. 301 For example, clusters 4 and 5 highlight a number of proteins that show increasing 302 abundance post-stress in the wild type cells, but are depleted in the mutant. These 303 highlight several biological processes that the mutant cells is no longer able to sustain in 304 the absence of one of the RAC Hsp70s, including much of nucleotide and amino acid 305 biosynthesis and metabolism.

306 Other functional enrichments are associated with the clusters, including the archetypal 307 HSR such as clusters 1 and 3 which include up regulated proteins involved in protein 308 folding and response to heat, and clusters 9 and 10 which are enriched for down regulated 309 proteins involved in ribosomal processing and protein synthesis.

\section{Identification of significantly changing protein profiles}

312 Significant protein fold changes across the time points were determined using the MSstats 313 package, adjusting $p$-values via the Benjamini \& Hochberg false discovery rate method ${ }^{46}$ 314 and also applying an empirical cutoff. Subsequently, 267 (WT) and 313 (ssb1 1 ) proteins 315 were determined to be significantly differentially abundant (see Methods) in at least one 316 time point during the 240 min time course, corresponding to $\sim 15 \%$ of all quantified 317 proteins. Only 78 proteins were found to be changing in both strains, a surprisingly small 318 overlap. However, those common changes were highly correlated between the two strains 319 in later time points. In particular, T0/T60 ratio in WT versus T0/T60 ratio in $s s b 1 \Delta$ 320 correlated; Spearman $=0.67$, p-value $<2.2 \times 10^{-16}$ and T0/T120; Spearman $=0.74, \mathrm{p}$ 321 value $<2.2 \times 10^{-16}$. This suggests a confined 'core' response that is essentially unaffected 322 by the deletion, supplementing a much greater strain-specific response likely to be linked 323 to the molecular function of the Ssb1 protein, as discussed later. Next, we focused our 324 analysis on the WT heat shock response.

325 Hallmark proteins of heat stress response (HSR)

326 The power of the proteomics approach becomes evident from its ability to pinpoint proteins 327 with well-known roles in heat stress. Among the proteins with most statistically significantly 328 increases in relative abundance in the WT were the small heat shock proteins with 329 chaperone activity, Hsp26p (adjusted p-value $=1.0 \times 10^{-16}$ ), the membrane associated 330 Hsp12p (adjusted p-value $=3.610^{-8}$ ) (Figure $3 \mathbf{A}$ ) as well as Hsp30p, all previously 
331 documented to be induced by heat stress $^{47-49}$. Interestingly, Hsp30p had no detectable 332 peptides and therefore no reported intensity in the T0 time point, but was quantified with 333 an average of 2 unique peptides in all other samples (Supplementary Figure 4). We were 334 therefore unable to calculate a fold change for Hsp30p, but based on the distribution of valid fold changes in the experiment we estimate it increases in abundance by at least 3336 fold in response to heat.

337 We also quantified both Hsp90 chaperone paralogs, Hsp82p and Hsc82p. Hsc82p was 338 moderately (>2-fold, adjusted p-value $2.4 \times 10^{-5}$ ) induced at T60, 120 and 240. 339 Comparatively, the Hsp82p isoform increased in abundance by over 3-fold two hours after 340 stress (adjusted p-value $5.5 \times 10^{-9}$ ) (Figure 3A). This data is in excellent agreement with 341 the transcriptome literature; HSP82 is strongly transcriptionally induced via the Hsf1p 342 factor, while HSC82 is expressed constitutively and also stimulated by Hsf1p, but 343 increases only marginally upon heat stress ${ }^{50,51}$.

344 Amongst the 1773 quantified proteins in the WT cells (Supplementary File 1), many that 345 displayed significant change and have not previously been associated with the HSR. Most notably, an uncharacterised protein YBR085C-A was induced up to eight-fold over the time course (adjusted p-value $1.4 \times 10^{-6}$ ) (Figure 3A). YBR085C-A has been shown to increase in response to DNA damage in a high-throughput study but has not previously been associated with heat stress response ${ }^{52}$. A PSI-BLAST search ${ }^{53}$ of YBR085C-A against the NCBI non-redundant database found no hits with annotated function. However, a literature search of known DNA-binding regulators revealed a number of transcription factors associated with the promoter region of this gene and thus likely regulating its expression. In particular, a study by Harbison et $a^{F^{4}}$ provided direct evidence of two heat shock transcription factors, Hsf1p and Msn2p, occupying the DNA regulatory sites of YBR085CA. The other transcription factors with experimental evidence of YBR085C-A DNA binding are displayed in Supplementary Table 1. More generally, we also note that up-regulated proteins at 240 minutes are significantly enriched for targets of several stress response transcription factors ${ }^{55}$ including Msn2p, Msn4p, Hsf1p (adjusted p-value $<1 \times 10^{-12}$ ) in all cases.

360 In contrast to widespread upregulation in the WT strain (211 out of the 267 differentially 361 abundant proteins), only 56 proteins exhibited a significant decrease in relative 362 abundance. For example, the most statistically significantly down-regulated proteins were 
363 Mup1p (adjusted p-value $\left.=1.1 \times 10^{-5}\right)$, Gas3p (adjusted p-value $=3.5 \times 10^{-10}$ ) and Pno1p 364 (adjusted $\mathrm{p}$-value $=3.1 \times 10^{-6}$ ) (Figure $\mathbf{3 A}$ ). The general trends in protein regulation 365 observed here were also recapitulated in other large-scale proteome studies; from the 366 Mann group ${ }^{26}\left(30\right.$ min incubation at $37^{\circ} \mathrm{C}$ ), and a study by Tyagi and Pedrioli ${ }^{56}$ (minimum 4 367 doublings at $37^{\circ} \mathrm{C}$ ). When considering the proteins reported as significantly changing in at 368 least one study, we observed positive, albeit weak pairwise correlation between the 369 relevant time points (inter-study Spearman correlations $0.44-0.62$, see Supplementary 370 Figure 5). This is typical of inter-lab correlations found in methodologically different 371 quantitative proteomic studies ${ }^{57}$. Full protein lists identified in this study are available in 372 Supplementary Files 2 and 3 for the WT and the ssb1 $\Delta$ samples.

373 Slow and sustained proteome remodelling in response to moderate heat stress

374 A distinguishing feature of our experimental strategy compared to previously reported 375 proteome studies is the temporal resolution of the dataset. The 10 and 30 minute time 376 points represent the early HSR, and 60, 120 and 240 minutes the mid to late response, 377 whereas other studies have considered longer times designed to reflect thermo-tolerance 378 adaptations (e.g. $16 \mathrm{~h}$ growth at $40^{\circ} \mathrm{C}$ by Shui \& Xiong et al. ${ }^{27}$ ). We sought to capitalise on 379 this temporal resolution and identify groups of proteins with different profiles across T0, 380 T10, T30, T60, T120 and T240 time points by performing c-means fuzzy clustering ${ }^{36,58}$.

381 Clustering analysis of both strains revealed only two major temporal profiles (Figure 3B); 382 proteins that were steadily increasing in abundance over time (cluster 1), and those that 383 were slowly decreasing (cluster 2). No dramatic shifts or transitory regulation in protein 384 expression was evident. This is in contrast to observations involving regulation of 385 phosphorylation in response to heat, where up to 15 clusters showing monotonous and 386 adaptation-like profiles could be identified ${ }^{59}$. A GO-term enrichment analysis revealed 387 relatively few significant terms within WT cluster 1 (Bonferroni-adjusted p-value $<0.05$ ) but 388 included expected terms such as protein refolding ( $p$-value $=2.2 \times 10^{-3}$, enrichment $=6.2$ ), 389 oligosaccharide metabolic processes ( $p$-value $=4.4 \times 10^{-2}$, enrichment $=5.3$ ) and cellular 390 response to heat $\left(p\right.$-value $=2.2 \times 10^{-3}$, enrichment $\left.=4.4\right)$. In contrast, cluster 2 proteins 391 displaying decreasing abundance (Figure 3B), had no significantly enriched GO-process 392 terms, consistent with non-specific degradation throughout the heat stress response. Full 393 GO enrichment data are provided in Supplementary File 4. 
394 A notable example of coordinated regulation in the heat stress response involved proteins 395 annotated with carbohydrate metabolism GO terms involved in the trehalose-synthesis 396 pathway. Trehalose is a storage disaccharide produced in yeast in a relatively simple metabolic pathway (Figure 4). It is a general stress protectant and contributes to the suppression of denatured protein aggregation. Following heat stress, the trehalosesynthesis protein (TPS) complex constituents (TPS1p, TPS2p, TPS3p, and TSL1p) were all significantly up-regulated; roughly 2 -fold after 120 minutes of heat, with the exception of TPS3p, a regulatory subunit of TPS, which showed much smaller changes (Figure 4). Similarly, the most abundant and highest affinity hexose transporter Hxt7p is up-regulated in preference to the others. These examples highlight the ability of proteomics to track the temporal molecular response in a coordinated fashion at the protein level across this pathway. We next turned our focus to the differences observed in the mutant strain.

ssb1 $\mathrm{HSR}$ is different to the wild type response

In the case of the ssb1 mutant strain that lacks this key member of the HSP70 family we observed notable differences in the membership of the clusters shown in Figure 3 and their associated gene ontology enrichments. Whilst clusters 1 and 2 retained the same overall trend in the two strains, a closer look at the dynamics of regulation and GO term analysis of the clusters reveals differences in their HSR response (Supplementary File 4). Whilst $s s b 1 \Delta$ cluster 1 retains proteins with functional enrichments in GO categories 'protein refolding', 'protein folding', and 'response to abiotic stimulus', the corresponding increases in relative protein abundance were generally not observed until later time points (T60 at earliest) and showed relatively small increases, between roughly 1.4 and 2 -fold change.

GO term enrichment analysis of cluster 2 (down-regulated) showed a very different picture in the mutant; 168 proteins were present in this cluster (compared to 48 in WT) with significant enrichment for cytoplasmic translation $\left(p\right.$-value $=1.8 \times 10^{-16}$, enrichment $\left.=4.4\right)$, 421 biogenesis/assembly $\left(p\right.$-value $=9.4 \times 10^{-8} / 1.1 \times 10^{-2}$, enrichment $\left.=2.6 / 3.7\right)$. This shows a more pronounced down-regulation of protein synthesis under stress. A direct comparison of the fold changes between WT and $s s b 1 \Delta$ at each time point highlights the differences in 424 the global response as well as in individual proteins in each strain (Supplementary Figure 425 6). This data confirms the trends captured by the clustering, we observed a general lack of 426 response in the mutant strain in the early time points (T0-T60), and an inability to up- 
regulate key proteins at the later time points (T120-T240). These molecular details are informative when contrasting with the phenotypic response observed in the absence of heat shock ${ }^{60}$, where deletion leads to only a modest decrease in fitness, consistent with our previous study ${ }^{61}$. Here, we can see how loss of a key member of the RAC leads to loss of ribosome and proteins involved in translation in general after stress in comparison to the WT response.

\section{Proteome, unlike transcriptome is not affected early in HSR}

The vast majority of the proteome did not display statistically significant changes in the early time points (T10 and T30). Even proteins with large differential regulation later in the experiment remained mostly unchanged T10-T30. This is in stark contrast to transcriptome-driven gene expression studies such as the seminal microarray study by Gasch et. al. ${ }^{9}$, that reported large mRNA changes within minutes of introduction of heat stress, mirrored in more recent studies by Strassburg et. al. ${ }^{62}$. There the authors measured mRNA changes in yeast cells exposed to heat stress in a time course experiment almost identical to ours. We decided to use this closely matched dataset to investigate the correlation between mRNA expression and protein abundance changes. To take full advantage of the time-resolved data, we performed global correlation analysis where a correlation coefficient is calculated based on all measurements at each time point. For the first analysis, we selected only the protein-transcript pairs for which a significant protein change was measured (resulting in 248 pairs).

Despite a strong correlation within experimental sub-types (transcriptome or proteome) and between time points, with $\mathrm{R}$ between $0.7-0.9$, we observed a poor global correlation between the transcriptome and proteome response $(R<0.3)$ during the early heat shock (Figure 5A). However, correlation increased globally over time, with the highest correlations $(R>0.45)$ being observed between the early transcript (15 to $60 \mathrm{~min})$ and late protein changes (120 to $240 \mathrm{~min}$ ), (Figure 5A). These findings are consistent with the immediate molecular response to elevated temperature, by regulating mRNA transcription via Hsf1p (but also potentially through post-translational modifications ${ }^{63}$ or modulating enzyme activities ${ }^{64}$ ). The attendant changes in protein level take longer to materialise due to the time it takes to synthesise the responding target proteins. This lag is further supported by the observation that expression of the majority of transcripts peaks between 
30 and 60 minutes, while most protein expression peaks 240 minutes after stress (Figure 5B). As visible in Figure 5B, the average delay in proteome response is estimated to be between 1 and 3 hours. However, the lack of fine-grained resolution makes it only a rough estimate. Furthermore, although the imperfect correlations between the transcriptome and proteome can most obviously be attributed to post-translational regulation, at least some of the variance is likely caused by measurement noise and technical differences between the two datasets.

\section{Global chaperone responses to the proteome under heat shock}

As chaperones are the principal molecular responders to heat shock, we examined their relative protein abundance changes across the time course (Figure 6), comparing the response in both the WT and mutant strain. This provides a unique picture of the global response for 60 quantified chaperones, in the context of their assigned classes following Gong et $\mathrm{al}^{41}$. Here, the added value of the temporal experiment is apparent, as consistent step-changes in relative abundance are obvious over time, rather than a simple "before/after" two time-point experiment that could be more prone to false positives. Clear patterns are apparent, highlighting how selected members of the Small chaperone family (Hsp12p, Hsp26p), Hsp60 and Hsp90s (Hsc82p, Hsp82p) all increase over the 240 minutes post-exposure. In parallel, selected Hsp70s increase in relative abundance to help maintain proteostasis. Notably, only a restricted set of chaperones are significantly increased in response to heat shock and indeed a few, as has been described before, decrease; Erj5p being the most obvious. This is consistent with its function as an endoplasmic reticulum-based transmembrane protein, where decrease or loss of function leads to a constitutive increase in the unfolded protein response ${ }^{65}$. A modest decrease in Ssb2p and also Ssb1p is also observed over 240 mins, genes that are also known to be down-regulated under heat stress ${ }^{11}$.

The global concerted heat shock response in wild type cells is not matched in the $s s b 1 \Delta$ mutant. Here, the global effects of Ssb1p absence are observed, leading to a marked attenuation of the main Hsp12p and Hsp26p increase and a generally weaker response across all the related heat shock factors. It is apparent that the loss of this key RAC associated Hsp70 prevents the cell from eliciting a full HSR, and it has knock-on effects on several areas of the chaperone network. For example, Mdj1 increases by 240 mins; Mdj1 is a mitochondrial Hsp40 that is highly connected in the chaperone network and involved in many key functions including folding of nascent peptides and degradation of misfolded 
492 proteins $^{66,67}$ - which are presumed to accrue at higher rates in the absence of Ssb1p 493 under heat stress. Ssa3p also shows a marked decrease at 240 mins and the Erj5p effect 494 is lost.

495 In parallel to the chaperones, we also examined attendant changes in their target 496 proteomes inferred from cognate interactions mapped by affinity-purification studies ${ }^{41}$, 497 shown on the right in Figure 6. A key feature here is the high correspondence between 498 chaperone abundance changes and their mapped target proteins, which display correlated 499 changes during the heat stress. For each chaperone, we display the fraction of their target 500 proteins present in the differentially abundant proteins (target rate, represented by circle 501 size in Fig 6), coloured by whether they largely increase (red) or decrease (green) in 502 relative abundance. The target rate reaches as high as $60 \%$ in some cases. We would not 503 expect to see $100 \%$ for several reasons. Firstly, chaperones are expected to attempt to 504 maintain proteostasis (i.e. maintain protein substrate levels) rather than increase them. 505 Therefore it is important to note we do not see a concerted decrease in relative abundance 506 for those proteins that are targets of the chaperones that increase in abundance. 507 Interestingly, chaperones also function by targeting misfolded clients for degradation 508 pathways, a route to a nominal decrease in abundance. However, we do not observe this 509 phenomenon directly in the quantitative proteomic data. Finally, shotgun proteomics 510 remains a stochastic technique that under-samples the proteome, resulting in some 511 undetected proteins from each chaperone target set. We also note that the data offers 512 strong support for the validity of the protein interactome data, with a direct demonstration 513 of how the specific targets of given chaperones are indeed protected from down-regulation 514 under a heat shock stress.

515 Figure 6 also shows how the targets of the chaperones in the mutant yeast strain are 516 affected, with a matched attenuation of the response to heat. Indeed, in several instances 517 the average level of some chaperone targets is notably reduced, such as those for Apj1p, 518 Ssb2p and most of the CCT complex. This highlights the widespread effects on the cellular 519 proteome of deleting a key member of the RAC complex. By 240 minutes, targets of all 520 three members of the remaining RAC proteins (Ssb2p, Zuo1p and Ssz1p) are reduced in 521 abundance, highlighting the 'sick' nature of the cells.

522 The nature of the global response can also be visualised by overlaying this quantitative 523 data on the chaperone-substrate protein interaction network of differentially abundant 
524 proteins, as shown in Figure 7. During the HSR, the chaperones and many of their 525 attendant targets increase in the WT cells. For example, after 10 minutes, an increase in 526 the highest-affinity glucose transporter Hxt7p is noted, followed by "small" group 527 chaperones after 30 minutes; by 60 minutes, a clear grouping of Hsp12p, Hsp26p, Hsp26p 528 and Hsp104p has formed, with attendant interconnected targets along with Hsp60p, both 529 HSP90s and selected cytosolic HSP70s. Targets of these chaperones including trehalose 530 biosynthetic enzymes Tps1p and Tsp2p are evident by 120 minutes along with the 531 chaperones Sse2p and a growing interlinked network of targets by 240 minutes. This 532 pattern is essentially replicated by the mutant strain but in a much-attenuated form (see 533 the lower half of Figure 7); an increase in Hsp26p levels is not detected at all and indeed 534 by 240 minutes two key HSP70s (Ssa3p and the other RAC-associate Ssb2p) are down535 regulated. This down-regulation of $\mathrm{Ssb} 2 \mathrm{p}$ affects many of its protein clients including 536 numerous ribosomal proteins.

537 The quantitative changes observed are also reflected in coherent common functional 538 responses (Figure 8, originally noted in Figure 2). Here, we observe global changes to the 539 entire functional network under heat stress, where the links between protein nodes are 540 coloured by common Gene Ontology (biological process) functional categories. Several 541 features are evident here over time, with expansion of the common protein clusters linked 542 to protein folding, response to heat and carbohydrate metabolic processes. Again, the 543 mutant strain exhibits an altered attenuated response pattern particularly in the earlier time 544 points with fewer proteins up-regulated. In contrast, by $240 \mathrm{~min}$ a substantial number of 545 proteins are reduced in abundance (green cirles), most notably linked to cytoplasmic 546 translation. This group is constituted by the majority of ribosomal proteins, which have 547 depressed abundance levels compared to the cells at T0.

\section{Discussion}

550 Budding yeast has been a model organism for elucidating the molecular mechanisms of 551 the heat shock response, most prominently, with global transcriptome profiling 8, 9, 25. 552 However, comprehensive time-resolved proteomics studies are so far not available. Here, 553 we addressed this gap and used single-shot LC-MS label-free quantitative proteomics to 554 elucidate both the initial response, and longer term general adaptation to mild heat stress. 555 In contrast to most previous heat shock studies, the shift from $30^{\circ} \mathrm{C}$ to $37^{\circ} \mathrm{C}$ investigated 
here is a smaller fluctuation in temperature. Accordingly, we observed subtle changes in protein expression, typically less than 1.5 fold, throughout the five time points sampled (T10, T30, T60, T120 and T240). This level of confident protein quantification was achieved through a robust normalisation strategy coupled to empirical cut offs derived from observed variance in WT:WT and $s s b 1 \Delta: s s b 1 \Delta$ signal. Nevertheless, some limitations inherent to all "relative quantification" experiments remain. For example, since we do not directly measure absolute protein abundances, it is not possible to disentangle the contribution of individual versus global synthesis rates to explain changes in relative protein abundance. Effectively, we measure the percentage contribution of each protein to the total cellular proteome and how this changes over time. We also note that a small lag is expected before the cells truly start experiencing the elevated temparature in our experiments, due to the time it takes for the media inside the culture flasks to equilibrate to the new temperature. Consequently, it makes the precise determination of the early time point kinetics difficult to assess accurately. Nevertheless, the amplitude of protein fold changes across the time-course of the experiment should not be significantly affected.

Our data confirm abundance changes in many hallmark HSR proteins, and for the first time, provides a detailed description of temporal patterns of expression for those proteins. In excellent agreement with the vast literature of heat shock response in $S$. cerevisiae ${ }^{1011}$, we detected a general down-regulation of proteins related to growth and protein synthesis, most notably ribosome biogenesis, and a widespread increase in abundance of stressrelated proteins ${ }^{47,68-70}$, including the Ssa family proteins whose modest up-regulation under mild heat stress is consistent with their role in preventing aggregation. However, there is a much greater up-regulation of small heat shock proteins, Hsp26p, Hsp12p, Hsp30p and Hsp42, coupled with a moderate induction of Hsp104p (1.5 fold 60 minutes after stress). This indicates that the cell puts greater emphasis on protein stabilisation by preventing aggregation and maintaining membrane and cytoskeletal organisation prior to kick-starting refolding and reactivation of the denatured proteins. We previously measured the absolute abundance changes in chaperone levels under heat shock using targeted mass spectrometry, with Hsp26p increasing to around $\sim 250,000$ copies per cell ${ }^{71}$. Hsp26 and Hsp42 are cytosolic chaperones that form oligomers and bind unfolded target proteins in order to stop them from aggregating. While Hsp42p is active in both normal and stress conditions, Hsp26p functions only in stress conditions ${ }^{70}$, even though both are regulated by Hsf1p and Msn2/Msn4p transcription factors ${ }^{72}$. Interestingly, proteins displaying 
589 significant abundance increases between 60-240 minutes were enriched for known targets 590 of several stress response transcription factors (Supplementary Figure 7), including 591 Msn2/4p, Hsf1p, Aft1p, Rpn4p. Rpn4p is the key regulator of proteasomal activity, which 592 as expected, is up-regulated to deal with a higher burden of protein degradation 593 associated with misfolded protein accumulation linked to heat shock. Rpn4p is also 594 coupled to other stress response transcription factors such as Pdr1p and Pdr3p, two zinc595 finger TFs that activate Rpn4 expression ${ }^{73}$. Although high-throughput shotgun proteomics 596 is not always sensitive enough to detect transcription factors, it is reassuring to be able to 597 detect the quantitative effects on their targets in a consistent manner.

598 Since chaperones perform multiple proteostatic roles, including disaggregation and 599 refolding, de novo folding of nascent polypeptides, and aiding degradation of misfolded 600 proteins, the increased folding workload under stress inevitably leads to increases in 601 abundance of the chaperone itself. In the wider context of established chaperone biology ${ }^{74}$, $602^{75}$, and the known effects of heat on the proteome (for example, protein denaturation and 603 aggregation) our results can be explained by two main scenarios leading to the overall 604 chaperone-target changes observed here. Scenario one, where proteins prone to 605 unfolding and aggregation in heat accumulate in the cell, but are protected by their 606 cognate chaperones from a general down-regulation, they manifest as relative increases in 607 protein abundance; Scenario two, where proteins (other than chaperones) that are 608 functionally important to counter the increase in temperature, such as trehalose 609 biosynthesis pathway components, are actively upregulated. In both cases, there is a need 610 to increase the amount of chaperone activity to either help refold the unfolded/misfolded 611 proteins or to help with de novo folding.

612 Our clustering of quantitative protein profiles in Figure 3 pointed to only two major 613 expression patterns present during heat stress response and minimal transient behaviour 614 was evident. More specifically, we did not detect many protein groups or biological 615 processes displaying complex temporal patterns above simple up- or down-regulation; we 616 did not observe a large set of proteins displaying any effective "recovery" step from the 617 stress. This general behaviour is different to that observed at the mRNA level. Gasch et 618 al. $^{9}$ showed an almost symmetrical transient response of mRNA up- and down-regulation. 619 Unsurprisingly, the time scale of protein response was also different. Gene expression 620 peaks within minutes of stress and returns to a new steady state within hours. These 621 dynamics were not observed for protein level changes, which required longer times to 
622 manifest and largely did not appear to return to basal levels. Although the stress and 623 growth conditions differ slightly between our proteomics and the Gasch mRNA studies ${ }^{9}$, 624 the same expression change trends were also observed in another study which integrated matching yeast proteomics and transcriptomics results when adapting to high osmolarity ${ }^{76}$. 626 The authors there also reported relatively large in amplitude and transient mRNA changes 627 and dampened protein levels for over a 1,000 transcript-protein pairs.

In addition to the WT heat shock experiment, we performed a corresponding study with cells lacking the Ssb1 chaperone. Although this protein is in fact down-regulated in heat shock, it is an interesting target due to its key role in nascent polypeptide folding. Indeed, as expected, our analyses show how its deletion exacerbates the effects of stress. The level of protein response exhibited in WT cells was lower and the abundance of most proteins did not change until late in the time course. ssb1 $\Delta$ cells do not appear to be capable of a strong heat stress response because the cells either do not sense the stimulus, are already pre-adapted to stress, or are unable to respond appropriately. The latter appears more likely, given the attenuated and delayed response we observe. For example, HSF1 targets continue to be over-represented in proteins up-regulated at 240 mins in the ssb1 cells (adjusted p-value $<4 \times 10^{-10}$ ) although only 16 are detected compared to 64 in the WT. Similarly, we note that Hsp12p and Hsp26p are themselves targets of Ssb1p (as well as many other chaperones), limiting the cells ability to deploy its full complement of stress response molecular repertoire. In the face of this inability to form an appropriate response, for example by producing more chaperones with de novo folding function, the cells have a limited RAC functionality. This strategy is consistent with the less protein production in general, and therefore less misfolded and aggregate-prone proteins in the cell. Curiously, less potential for aggregation would reduce the need for expressing stabilising proteins like Hsp12p, Hsp26p.

647 Previously, we have shown that cells deleted for Ssb1p are able to maintain protein homeostasis and do not undergo major proteome remodelling in normal conditions ${ }^{28}$. We proposed that this is possible owing to other HSP70 chaperones taking over the folding duties of Ssb1p and spreading the workload across the network. Here, we suggest that 651 under heat stress the system is pushed to breaking point leading to a collapse in the 652 requisite chaperone and protein synthetic systems, epitomised by the systemic downregulation of ribosomal proteins shown in Figure 8 after 240 minutes. 
655 More generally, our results demonstrate the power of quantitative proteomics coupled to 656 integrated bioinformatics to inform on the molecular events underpinning a cellular 657 response to stress in a temporal fashion. Additionally, by consideration of a deletant strain 658 in parallel, the global consequences of the loss of that gene on the ability of the cell to 659 respond to stress may be characterised and used to rationalise the molecular deficiencies 660 that lead to phenotypes.

661 Overall, the results presented in this study provide a detailed description of the heat shock 662 response in $S$. cerevisiae at the protein level, integrating this information with gene 663 expression changes. This analysis allows us to examine expression patterns of individual 664 proteins in fine detail, and to evaluate the more holistic global response, uncovering 665 important trends on a system level. Finally, the data obtained here are in excellent 666 agreement with the vast, mainly transcriptome-based literature of heat shock response, 667 but emphasises that novel insights can still be gained from carefully controlled large-scale 668 quantitative proteomics studies that cannot be deduced by examining the transcriptome 669 alone.

670

671 Acknowledgements

672 The authors acknowledge support from the Biotechnology and Biological Sciences 673 Research Council in the form of a studentship to AFJ as part of the Systems Biology 674 Doctorial Training Centre, and grant support to SJH, CMG and CEE in the form of a LoLa 675 grant (BB/G009112/1), and additional BBSRC support for MGA (BB/M025748/1).

\section{Conflicts of interest}

678 There are no competing or conflicting interests from any of the authors. 


\section{REFERENCES}

1. D. J. Barshis, J. T. Ladner, T. A. Oliver, F. O. Seneca, N. Traylor-Knowles and S. R. Palumbi, Proc. Natl. Acad. Sci. U. S. A., 2013, 110, 1387-1392.

2. K. W. Kim and Y. Jin, FEBS Lett., 2015, 589, 1644-1652.

3. E. de Nadal, G. Ammerer and F. Posas, Nat. Rev. Genet., 2011, 12, 833-845.

4. R. C. Taylor, K. M. Berendzen and A. Dillin, Nat. Rev. Mol. Cell Biol., 2014, 15, 211-217.

5. H. Karathia, E. Vilaprinyo, A. Sorribas and R. Alves, PLoS One, 2011, 6, 10.

6. S. Hohmann, Microbiol. Mol. Biol. Rev., 2002, 66, 300-+.

7. S. M. O'Rourke, I. Herskowitz and E. K. O'Shea, Trends Genet., 2002, 18, 405-412.

8. H. C. Causton, B. Ren, S. S. Koh, C. T. Harbison, E. Kanin, E. G. Jennings, T. I. Lee, H. L. True, E. S. Lander and R. A. Young, Molecular Biology of the Cell, 2001, 12, 323-337.

9. A. P. Gasch, P. T. Spellman, C. M. Kao, O. Carmel-Harel, M. B. Eisen, G. Storz, D. Botstein and P. O. Brown, Molecular Biology of the Cell, 2000, 11, 4241-4257.

10. K. A. Morano, C. M. Grant and W. S. Moye-Rowley, Genetics, 2012, 190, 1157-1195.

11. J. Verghese, J. Abrams, Y. Y. Wang and K. A. Morano, Microbiol. Mol. Biol. Rev., 2012, 76, 115-158.

12. K. Richter, M. Haslbeck and J. Buchner, Mol. Cell, 2010, 40, 253-266.

13. M. B. Eisen, P. T. Spellman, P. O. Brown and D. Botstein, Proc. Natl. Acad. Sci. U. S. A., 1998, 95, 14863-14868.

14. P. K. Sorger and H. R. B. Pelham, Cell, 1988, 54, 855-864.

15. G. Wiederrecht, D. Seto and C. S. Parker, Cell, 1988, 54, 841-853.

16. J. S. Hahn, Z. Z. Hu, D. J. Thiele and V. R. Iyer, Mol. Cell. Biol., 2004, 24, 5249-5256.

17. E. J. Solis, J. P. Pandey, X. Zheng, D. X. Jin, P. B. Gupta, E. M. Airoldi, D. Pincus and V. Denic, Mol Cell, 2016, 63, 60-71.

18. A. P. Schmitt and K. McEntee, Proc. Natl. Acad. Sci. U. S. A., 1996, 93, 5777-5782.

19. N. Lopez, J. Halladay, W. Walter and E. A. Craig, J. Bacteriol., 1999, 181, 3136-3143.

20. C. Pfund, N. Lopez-Hoyo, T. Ziegelhoffer, B. A. Schilke, P. Lopez-Buesa, W. A. Walter, M. Wiedmann and E. A. Craig, Embo J., 1998, 17, 3981-3989.

21. R. J. Nelson, T. Ziegelhoffer, C. Nicolet, M. Wernerwashburne and E. A. Craig, Cell, 1992, 71, 97-105.

22. M. Gautschi, H. Lilie, U. Funfschilling, A. Mun, S. Ross, T. Lithgow, P. Rucknagel and S. Rospert, Proc. Natl. Acad. Sci. U. S. A., 2001, 98, 3762-3767.

23. M. Rakwalska and S. Rospert, Mol. Cell. Biol., 2004, 24, 9186-9197.

24. K. Peisker, M. Chiabudini and S. Rospert, Biochim. Biophys. Acta-Mol. Cell Res., 2010, 1803, 662672.

25. K. Strassburg, D. Walther, H. Takahashi, S. Kanaya and J. Kopka, Omics, 2010, 14, 249-259.

26. N. Nagaraj, N. A. Kulak, J. Cox, N. Neuhauser, K. Mayr, O. Hoerning, O. Vorm and M. Mann, Mol. Cell. Proteomics, 2012, 11, 11.

27. W. Q. Shui, Y. Xiong, W. D. Xiao, X. N. Qi, Y. Zhang, Y. P. Lin, Y. F. Guo, Z. D. Zhang, Q. H. Wang and Y. H. Ma, Mol. Cell. Proteomics, 2015, 14, 1885-1897.

28. A. F. Jarnuczak, C. E. Eyers, J. M. Schwartz, C. M. Grant and S. J. Hubbard, Proteomics, 2015, DOI: 10.1002/pmic.201400527.

29. J. Cox and M. Mann, Nat. Biotechnol., 2008, 26, 1367-1372.

30. J. Cox, N. Neuhauser, A. Michalski, R. A. Scheltema, J. V. Olsen and M. Mann, J. Proteome Res., $2011,10,1794-1805$.

31. J. Cox, M. Y. Hein, C. A. Luber, I. Paron, N. Nagaraj and M. Mann, Mol. Cell. Proteomics, 2014, 13, 2513-2526.

32. A. I. Nesvizhskii and R. Aebersold, Mol. Cell. Proteomics, 2005, 4, 1419-1440.

33. O. Serang and W. Noble, Stat. Interface, 2012, 5, 3-20.

34. J. A. Vizcaino, E. W. Deutsch, R. Wang, A. Csordas, F. Reisinger, D. Rios, J. A. Dianes, Z. Sun, T. Farrah, N. Bandeira, P. A. Binz, I. Xenarios, M. Eisenacher, G. Mayer, L. Gatto, A. Campos, R. J. Chalkley, H. J. Kraus, J. P. Albar, S. Martinez-Bartolome, R. Apweiler, G. S. Omenn, L. Martens, A. R. Jones and H. Hermjakob, Nat. Biotechnol., 2014, 32, 223-226. 
35. M. Choi, C. Y. Chang, T. Clough, D. Broudy, T. Killeen, B. MacLean and O. Vitek, Bioinformatics, 2014, 
65. M. Carla Fama, D. Raden, N. Zacchi, D. R. Lemos, A. S. Robinson and S. Silberstein, Biochim Biophys Acta, 2007, 1773, 232-242.

66. I. Wagner, H. Arlt, L. van Dyck, T. Langer and W. Neupert, EMBO J, 1994, 13, 5135-5145.

67. P. Walsh, D. Bursac, Y. C. Law, D. Cyr and T. Lithgow, EMBO Rep, 2004, 5, 567-571.

68. M. Wernerwashburne, J. Becker, J. Kosicsmithers and E. A. Craig, J. Bacteriol., 1989, 171, 26802688.

69. W. R. Boorstein and E. A. Craig, J. Biol. Chem., 1990, 265, 18912-18921.

70. M. Haslbeck, N. Braun, T. Stromer, B. Richter, N. Model, S. Weinkauf and J. Buchner, Embo J., 2004, 23, 638-649.

71. R. J. Mackenzie, C. Lawless, S. W. Holman, K. Leteomics, 2016, 16, 2128-2140.

72. M. Amoros and F. Estruch, Mol. Microbiol., 2001, 39, 1523-1532. 


\section{Figures}

803 Figure 1 Overview of proteomics strategy and label free quantification results. Yeast cultures (WT and ssb1 $\triangle$ ) were grown at $30^{\circ} \mathrm{C}$ to mid exponential phase before transfer to $37^{\circ} \mathrm{C}$, and subsequent sampling at $0,10,30,60$, 120 and 240 minutes; the entire experiment was performed in four biological replicates. Cell extracts for each strain were prepared and protein digests analysed by LC-MS/MS using an LTQ-Orbitrap Velos. Mass spectrometry data was acquired for 24 WT and 24 ssb1 $\Delta$ samples in two separate experimental blocks. B) Left panel: Venn diagram shows the overlap of the number of quantified proteins in WT and ssb1 $\Delta$ strains. Middle panel: two boxplots display the number

A
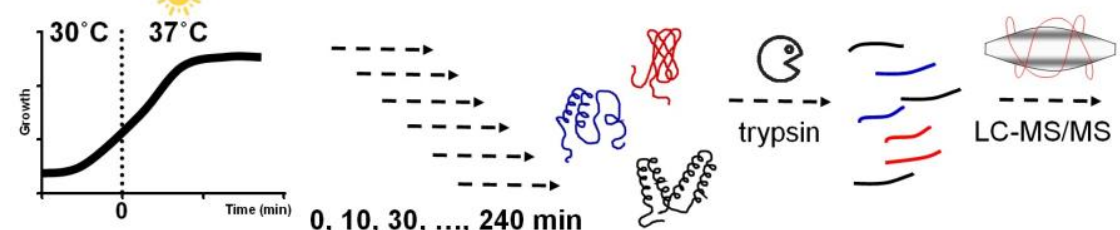

$0,10,30, \ldots, 240 \mathrm{~min}$

B
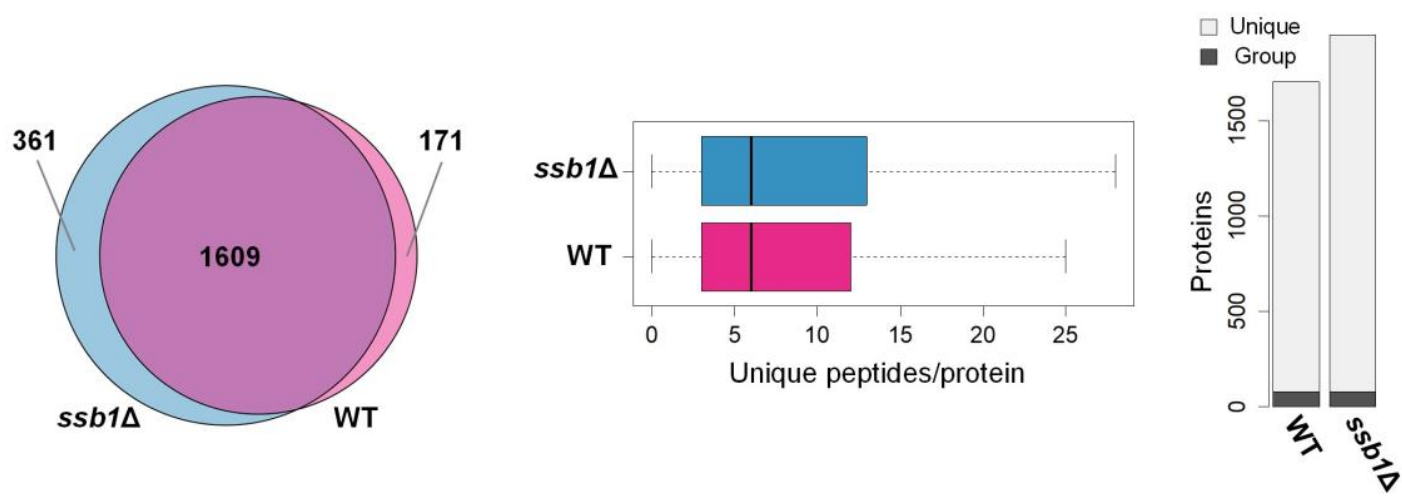
Figure 2 Heatmap and functional enrichment of temporal protein profiles during HSR. Heat map visualisation of the entire quantitative proteomic profiles under the heat shock response in WT and ssb1 $\triangle$. At each time point (column) $\log _{2}$ ratio to time 0 is plotted. Each row represents a protein and rows were clustered using Euclidean distance using all timepoints, so that similarities in gene profiles between the two strains can be observed. This is evident from the coherent clusters which display common Gene Ontology functional enrichments (See Supplementary File 4) based on GO Slim. Clusters display both common (e.g. cluster 1 ) and opposed (e.g. cluster 5) expression patterns between WT and ssb1 $\Delta$ strains.

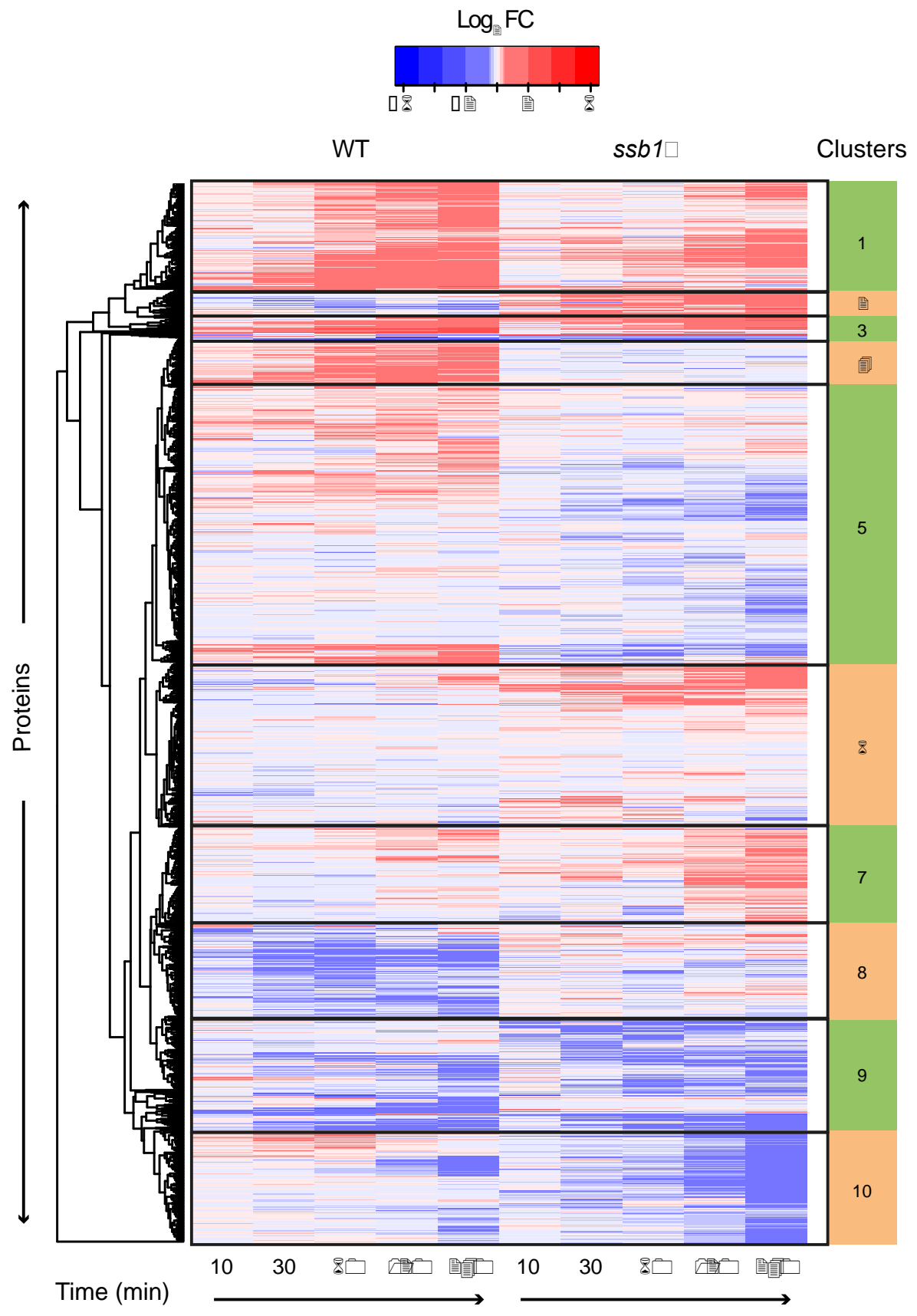


Figure 3. Temporal protein profiles after heat shock stress in WT and ssb1 $\mathbf{\Delta}$ cells. A) Example protein profiles for well-established heat stress responders with most significant up-regulation (Hsp26, Hsp12, Hsp82), as well as YBR085C-A, the highest induced uncharacterized protein. Gas3 and Pno1p are examples of the most significantly repressed proteins. Red circles signify statistically significant expression change at any given time point, and the size of the circle represents significance (bigger is more significant). B) Unsupervised clustering of temporal protein profiles. Two dominant clusters were obtained using fuzzy cmeans algorithm for both WT and ssb1 $\Delta$ cells, displaying proteins with membership greater than 0.7 (210 and 48 proteins in clusters 1 and 2 in WT, and 140 and 168 in the ssb1 $1 \Delta$ mutant strain). Notably, more proteins display the cluster 2 "decreasing" abundance profile in the mutant strain.

A
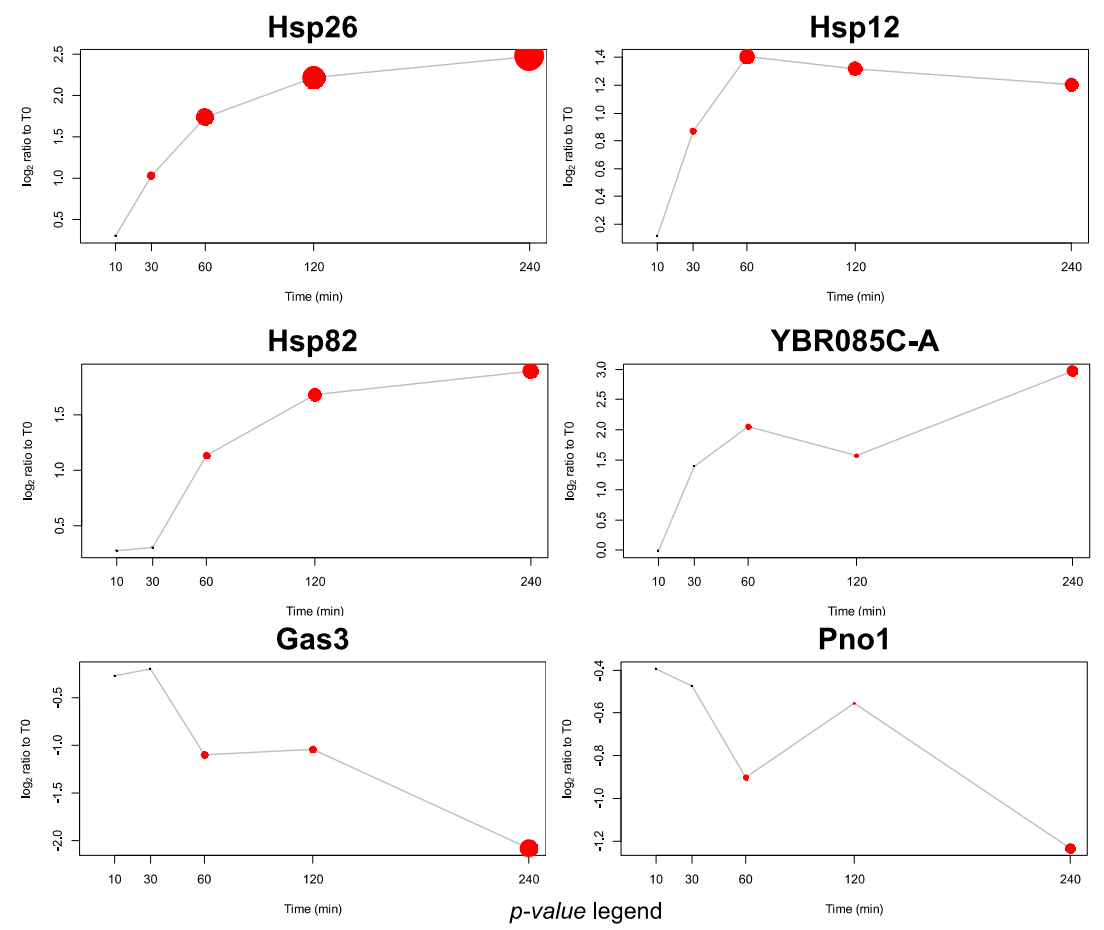

$\begin{array}{ccc} & 1.5-09 & 0.0005 \\ - & 58-07 & 0.005 \\ 58-05 & : & 0.05 \\ - & >0.05 \text { (not significa }\end{array}$

B
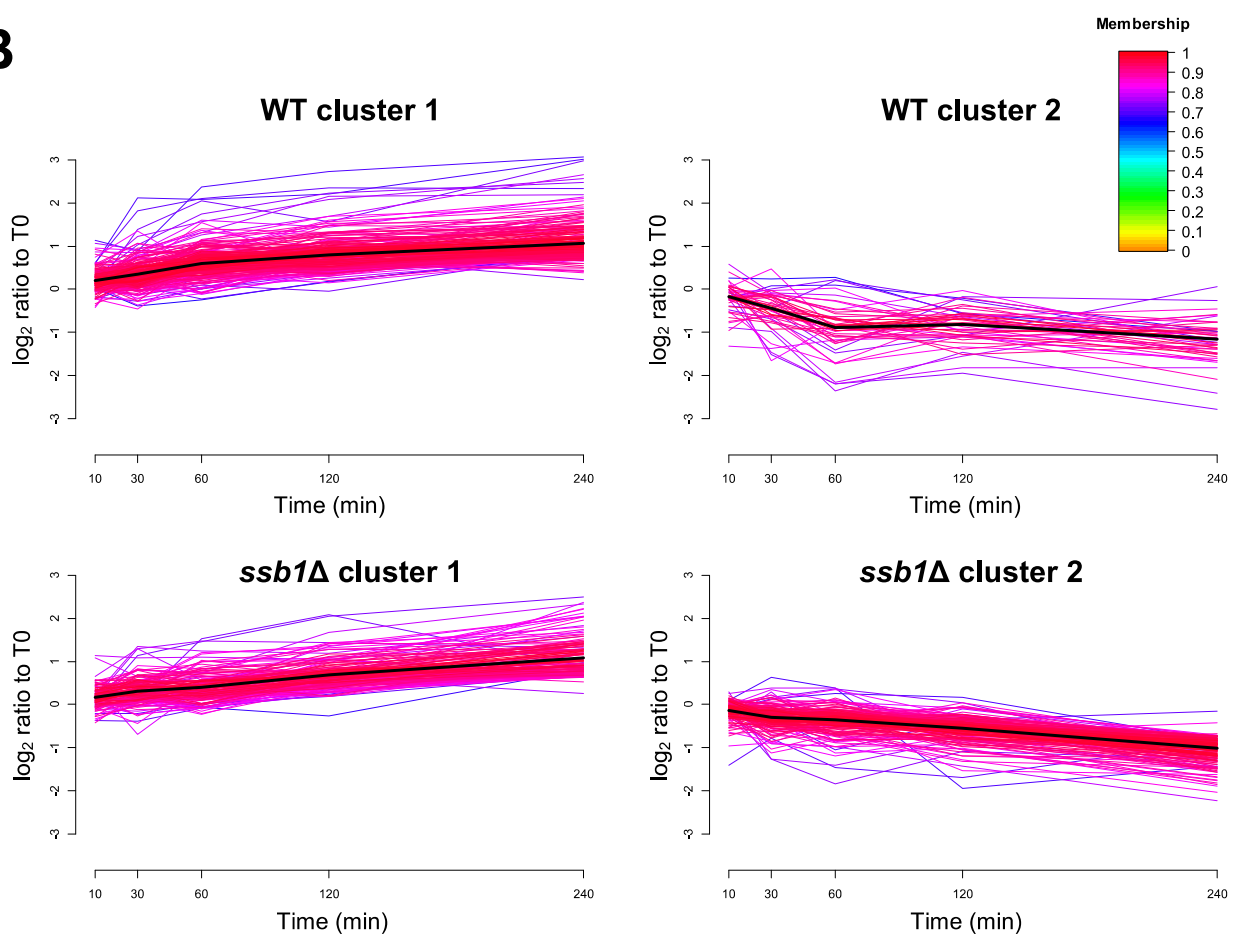
833 Figure 4. Heatshock induced proteome changes in the trehalose biosynthetic pathway. A bubble834 plot overlaid on the trehalose pathway displaying relative protein abundance changes under heat 835 stress of key enzymes. Red indicates abundance increases, green a decrease, with circle size 836 corresponding to statistical significance of the changes. The increases are typically over 2-fold over unstressed cells after 240 minutes in WT cells, but this is attenuated in the ssb1 $\Delta$ mutant.

838

\section{Trehalose metabolism}

WT

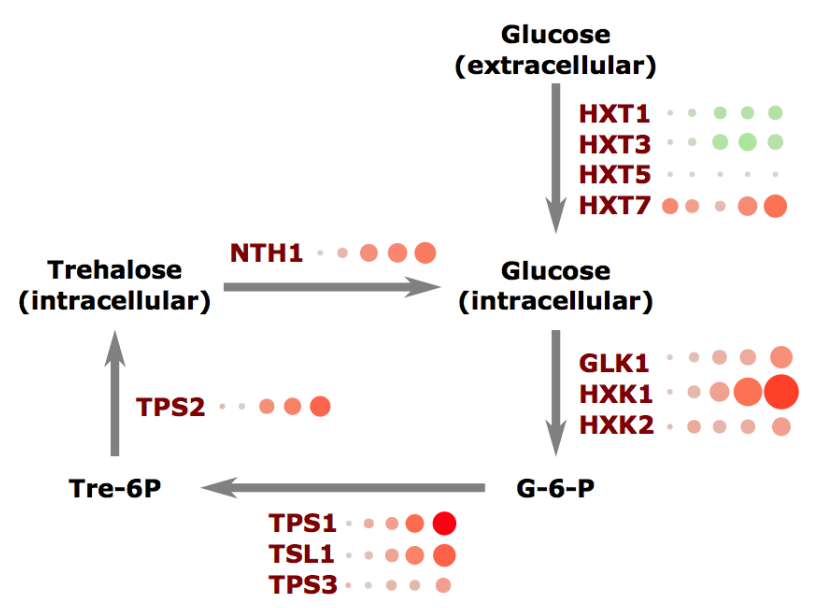

$\log _{2}$ FC

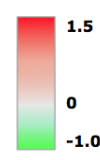

$\log _{10}$ Adj-pvalue

- 0

4
8

$\begin{aligned} & 12 \\ & 16\end{aligned}$

839

840

841

842 

quantitative proteomic profiles, comparing relative expression changes at individual time points. The upper and right-hand quadrants compare intra-technique expression profiles showing high correlations between transcriptomes, and between proteomes, and lower correlations when transcriptomes and proteomes are compared. The highest correlations between transcriptome and proteome are indicated on the plot; a time lag is also evident between eary and late time points. B) Histograms showing the number of differentially expressed transcripts and proteins whose expression peaks at each given time point with respect to unstressed cells at time zero. Protein expression is still rising in differentially expressed genes at 240

A

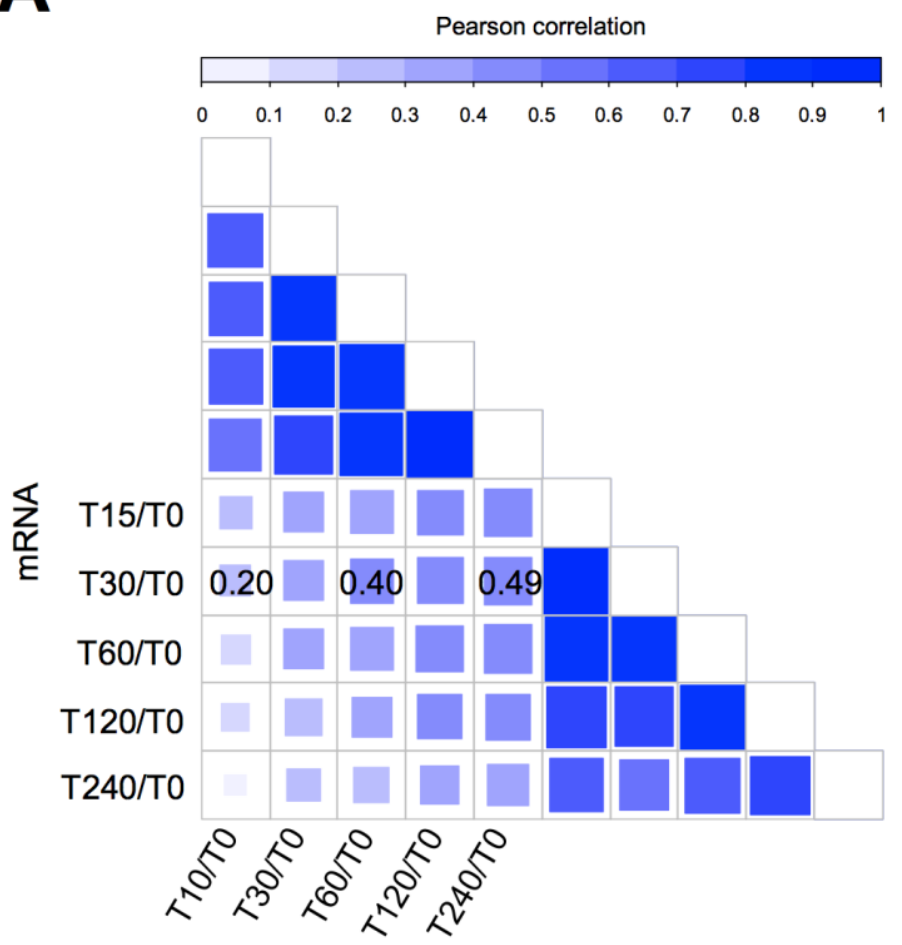

Protein
B

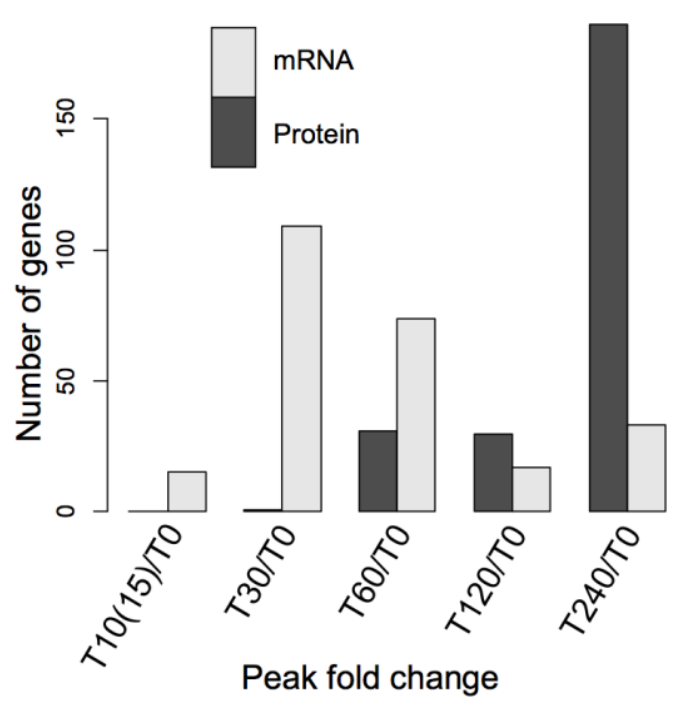


Figure 6. Global proteome changes in the yeast chaperome under heat stress. Left: The bubble-plot shows protein abundance for all chaperones, in both wild type and mutant cells, across the 240-minute time course. Red circles indicate increasing protein abundance changes according to the key, with green indicating decrease. The full set of 60 chaperones detected in the label-free mass spectrometry data are shown, indicating a localised response from a limited set of chaperones, chiefly in the Small class. Right: The panel shows the corresponding changes in the annotated client proteins, showing the relative fraction of chaperone-specific targets detected in the differentially abundant protein set at that time point (adjusted $\mathrm{p}<0.05$ ), i.e. the "Target Rate". Circles are coloured by the average abundance change (red increasing, green decreasing). Protein substrates follow the trends of their parent chaperones. The changes in both the chaperones and their targets are attenuated for the mutant strain.

\section{Chaperone proteins}

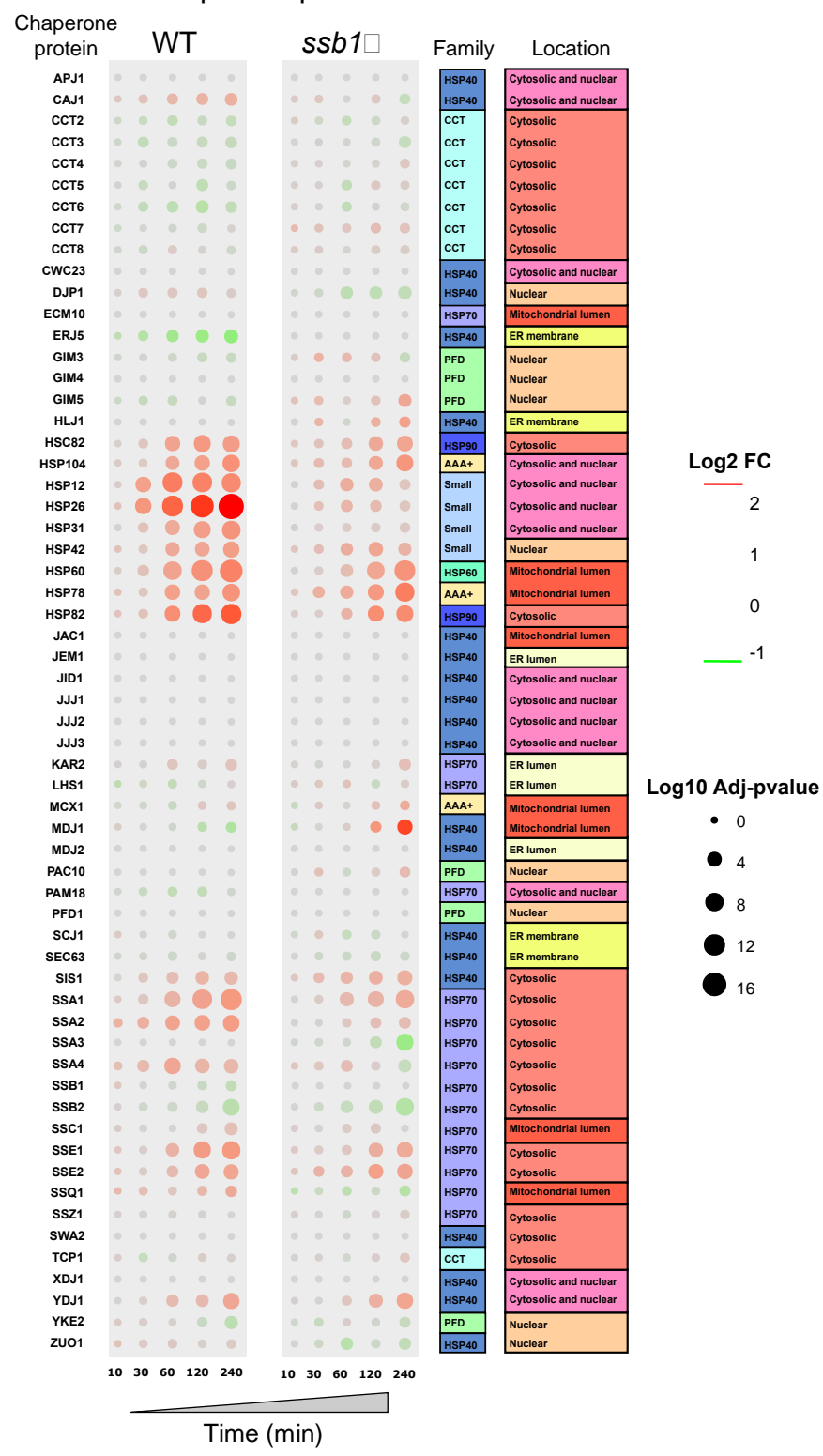

Target proteins

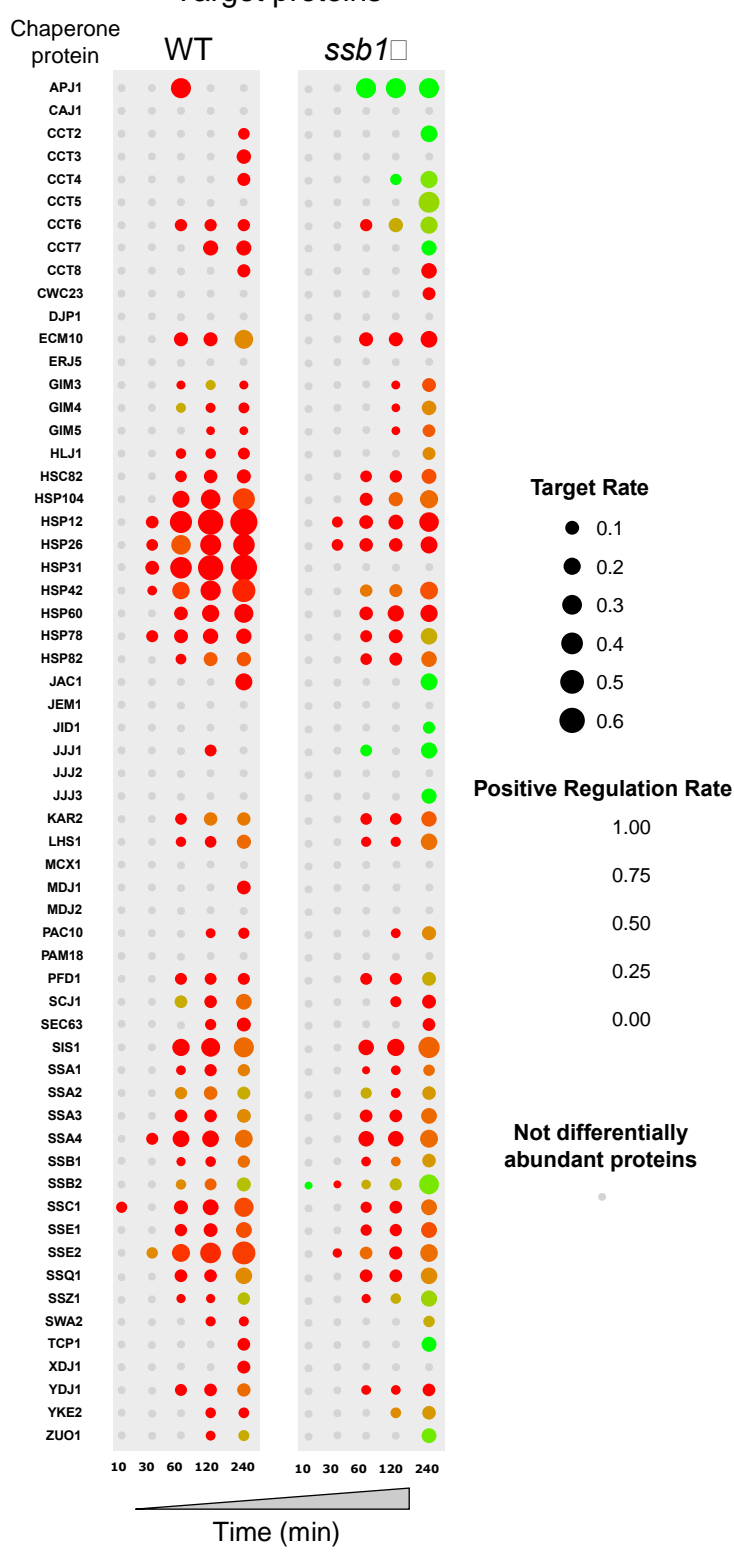


870 Figure 7. Temporal changes to the yeast chaperone-linked protein interaction network under heat shock. 871 Proteins displaying differential abundance are shown as nodes in the graph, linked to cognate protein 872 substrates via edges. Only chaperones or chaperone targets displaying significant abundance changes at 873 each time point are shown, with WT cells in the upper panel and ssb1 $\Delta$ mutant in the lower panel.

874

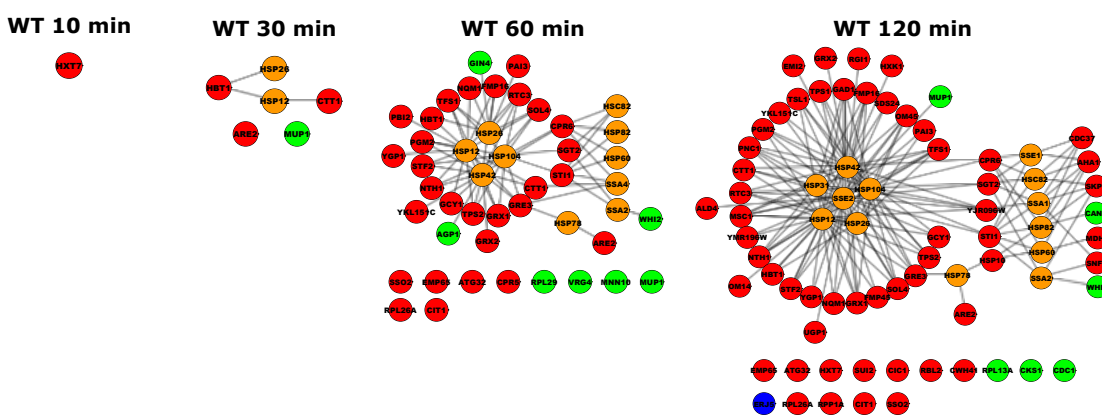

$s s b 1 \triangle 10 \mathrm{~min}$

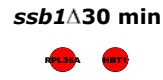

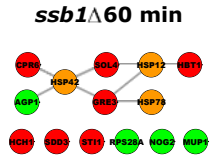

Up regulated differentially abundant target protein Down regulated differentially abundant target protein Up regulated differentially abundant chaperone protein
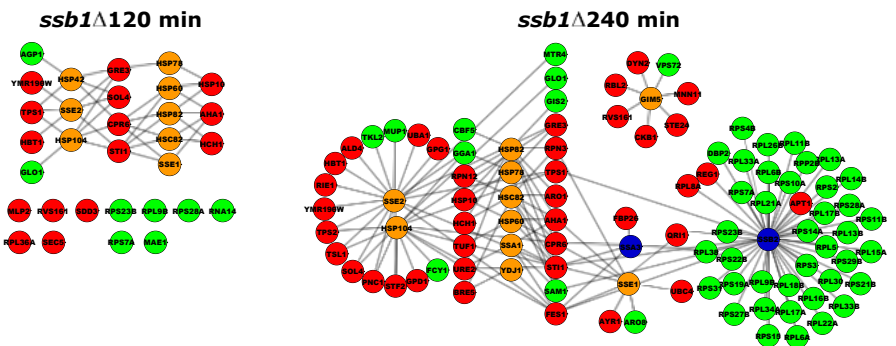

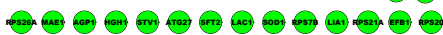

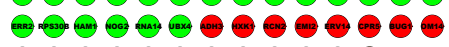

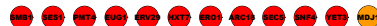


Figure 8. Functional network changes to the proteome during heat shock. Differentially abundant proteins during the heat shock response are shown as nodes in the two graphs (WT on the left, and ssb1 $\Delta$ mutant on the right), with edges linking proteins that share common Gene Ontology biological process terms. The growth of the heat shock response is apparent as concerted blocks of common function expand over time, notably involved in carbohydrate metabolism, protein folding and response to heat. Equally, the ability of the mutant ssb1 $\Delta$ strain to respond is evident, particularly in complete down-regulation of cytoplasmic

883 translation capacity as almost all ribosomal proteins are decreased in abundance by 240 minutes.

884
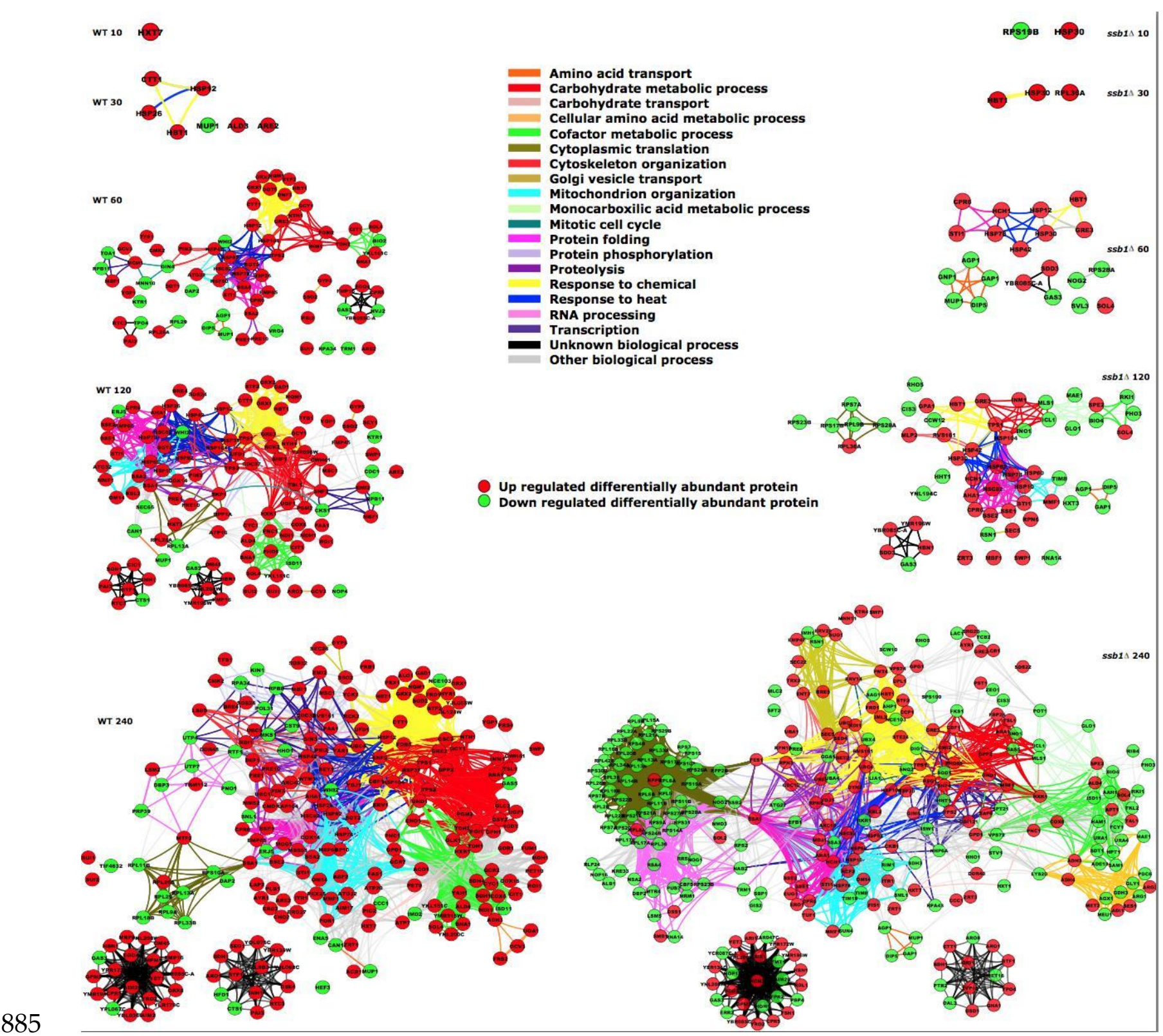


\section{Supplementary Figures and Tables}

\section{A QUANTITATIVE AND TEMPORAL MAP OF PROTEOSTASIS DURING HEAT SHOCK IN SACCHAROMYCES CEREVISIAE}

Andrew F. Jarnuczak ${ }^{1,3}$, Manuel Garcia Albornoz ${ }^{1}$, Claire E. Eyers ${ }^{2}$, Christopher M. Grant ${ }^{1}$, Simon J. Hubbard ${ }^{1 *}$

4. School of Biological Sciences, Faculty of Biology, Medicine and Health, University of Manchester, Manchester Academic Health Science Centre, Oxford Road, Manchester M13 9PT

5. Centre for Proteome Research, University of Liverpool, Department of Biochemistry, Institute of Integrative Biology, Liverpool, L69 7ZB, UK

6. European Bioinformatics Institute (EMBL-EBI), Wellcome Trust Genome Campus, Hinxton, Cambridge, United Kingdom

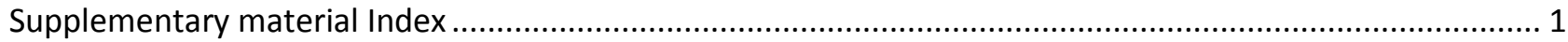

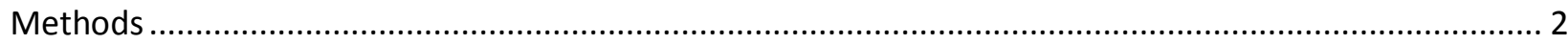

Detailed description of protein significance testing procedure ..................................................... 2

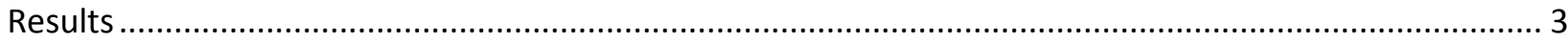

Supplementary Table 1.Transcription factors with experimental evidence of binding to YBR085C-A .... 3

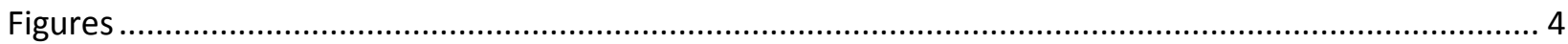

Supplementary Figure 1 Evaluation of WT and ssb1 $\Delta$ cell growth before and during heat stress. ........ 4

Supplementary Figure 2 Quantification repeatability from mass spectrometry acquisition................. 5

Supplementary Figure 3 Definition of protein fold change thresholds. ............................................ 6

Supplementary Figure 4 Quantification of Hsp30p in the WT samples. ........................................... 7

Supplementary Figure 5 Correlation of protein fold changes between this and two other yeast heat

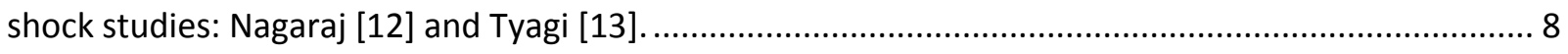

Supplementary Figure 6. Comparison of protein abundance fold changes between wold type and mutant yeast strains at matched time points after heat shock. ................................................. 9

Supplementary Figure 7. Enrichment of transcription factor targets in the upregulated proteome over

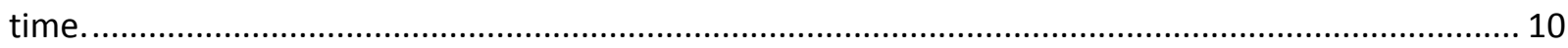

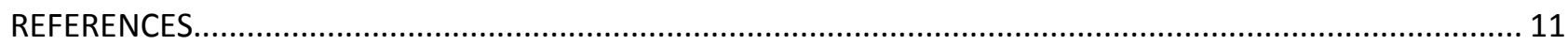




\section{Methods}

\section{Detailed description of protein significance testing procedure}

In order to determine statistically significantly changing proteins with respect to time point T0 we used the MSstats package [1]. Protein identities, conditions, biological replicates and intensities from MaxQuant were uploaded, retaining protein ID information from the 'proteinGroups.txt' file, conditions and biological replicates from the 'annotation.csv' file, and intensities from the 'evidence.txt' file. Data normalization was performed using the 'equalizeMedians' option and summarization using the 'Tukey's median polish' option. Following this, a condition comparison was performed using the 'groupComparison' option. $\log _{2}$ fold changes and adjusted $p$ values were obtained for the WT and ssb1 $\Delta$ strains. .

Additionally, we defined empirical thresholds for the minimum magnitude of change a protein had to display in order to be considered significantly regulated. This was based on the technical variance in each block of LC-MS analysis. To that end, a distribution of associated fold changes was generated by calculating ratios between all permutations of the QC sample pairs in either WT-QC or ssb1 $1-Q C$ (e.g. QC1 vs QC2, QC1 vs QC3, ..., QC2 vs QC3, etc.) (Supplementary Figure 3). The $2.5^{\text {th }}$ and $97.5^{\text {th }}$ percentile values of the resulting Gaussian distributions were then assigned as down- and up-regulation thresholds respectively. The thresholds were $0.69<\log 2 \mathrm{FC}<-0.958$ for the WT strain and $0.634<\log 2 \mathrm{FC}<-0.714$ for the ssb1 $\Delta$ mutant. Only proteins with fold changes outside these thresholds and an associated FDR corrected $p$-value $<0.05$ were considered significantly changing. 


\section{Results}

Supplementary Table 1.Transcription factors with experimental evidence of binding to YBR085C-

A

\begin{tabular}{|c|c|c|}
\hline $\begin{array}{c}\text { Transcription } \\
\text { Factor }\end{array}$ & Study & Experiment type \\
\hline Hsf1p & Harbison et al. [2] & ChIP-on-chip \\
\hline Ino4p & Workman et al. [3] & ChIP-on-chip \\
\hline Msn2p & Harbison et al. [2] & ChIP-on-chip \\
\hline Pho2p & Harbison et al. [2] & ChIP-on-chip \\
\hline Skn7p & Harbison et al. [2] and Ni et al. [4] & ChIP-on-chip \\
\hline Sok2p & Borneman et al. [5] & ChIP-on-chip \\
\hline Fhl1p & Kasahara et al. [6] & ChIP-on-chip \\
\hline Sko1p & Capaldi et al. [7] and Ni et al. [4] & ChIP and ChIP-on- \\
& & chip \\
\hline Ste12p & Lefrancois et al. [8] & ChIP-seq \\
\hline Yap1p & Tan et al. [9] & ChIP-on-chip \\
\hline Gcn4p & Ernst et al. [10] & ChIP-on-chip \\
\hline Hmo1p & Kasahara et al. [6] & ChIP-on-chip \\
\hline Cin5p & Ni et al. [4] & ChIP-on-chip \\
\hline Yap6p & Ni et al. [4] & ChIP-on-chip \\
\hline Abf1p & ChIP-on-chip \\
\hline
\end{tabular}




\section{Figures}

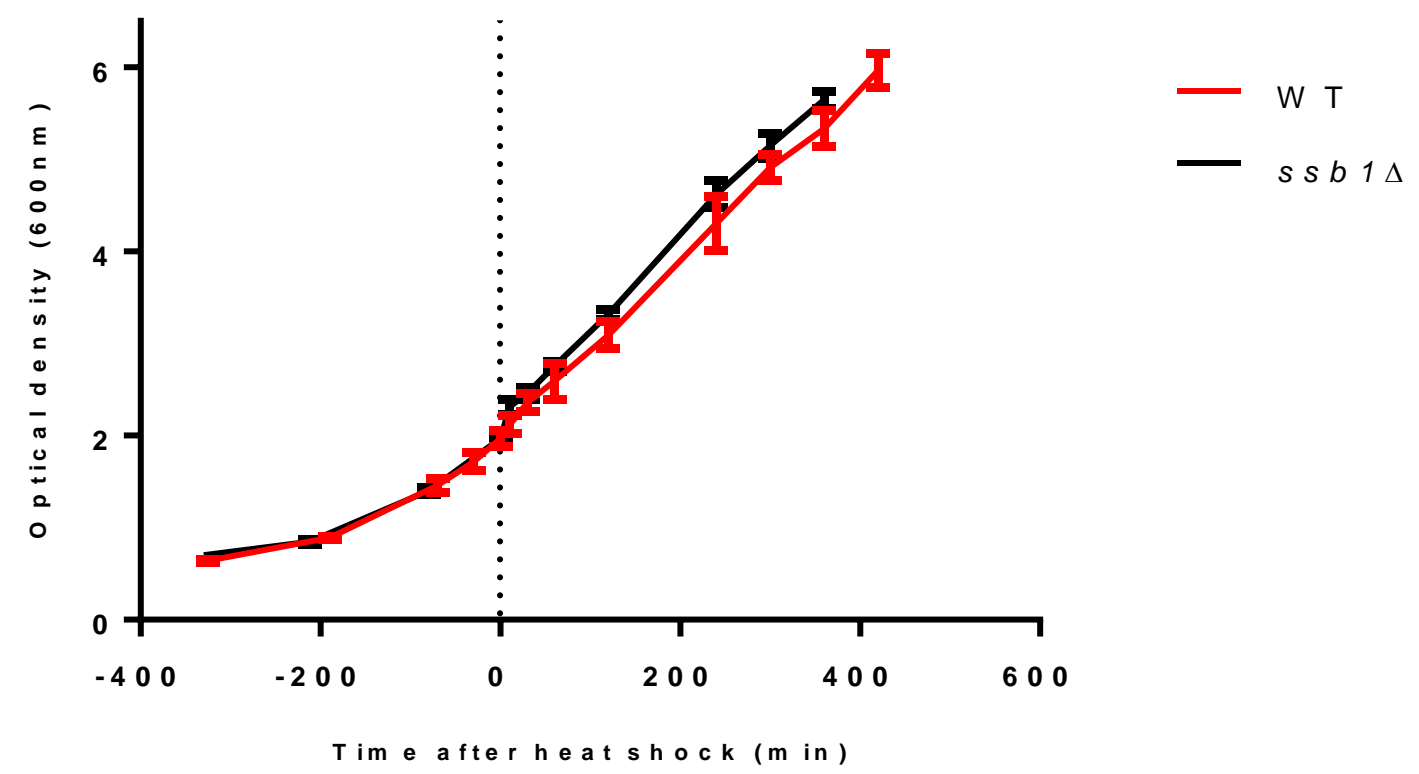

Supplementary Figure 1 Evaluation of WT and $\operatorname{ssb} 1 \Delta$ cell growth before and during heat stress.

Optical density (at $600 \mathrm{~nm}$ ) of the cultures was measured and results are shown as the mean \pm standard deviation of the four biological replicates. Dashed line indicates transfer from $30^{\circ} \mathrm{C}$ to $37^{\circ} \mathrm{C}$. 


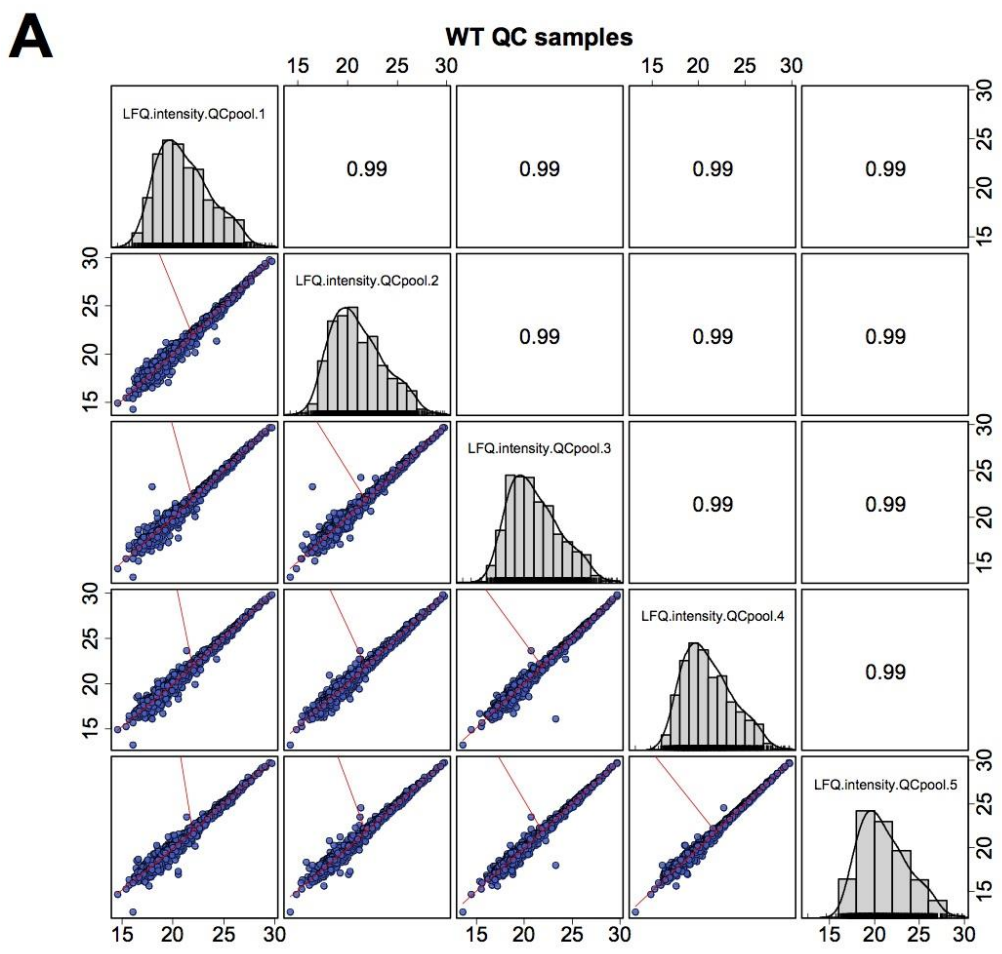

B

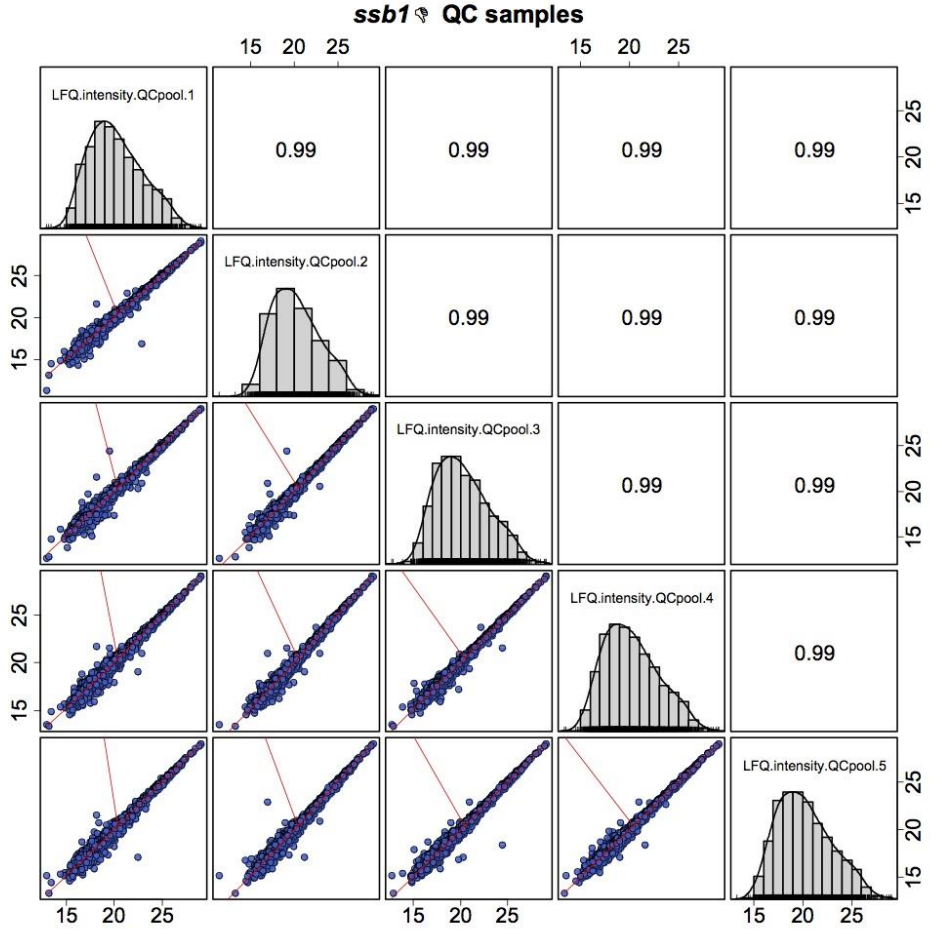

\section{Supplementary Figure 2 Quantification repeatability from mass spectrometry acquisition.}

The scatterplot matrix below the diagonal shows a comparison of label free protein quantifications ( $\log 2)$ between the five $\mathrm{QC}$ samples acquired through the mass spectrometry run. On the diagonal, histograms of intensity distributions in each $\mathrm{QC}$ sample are displayed. The number shown above the diagonal is the Pearson correlation coefficient between the two relevant replicates. B) A corresponding scatterplot for SSB1 data. 


\section{Histogram of all WT QC ratios}

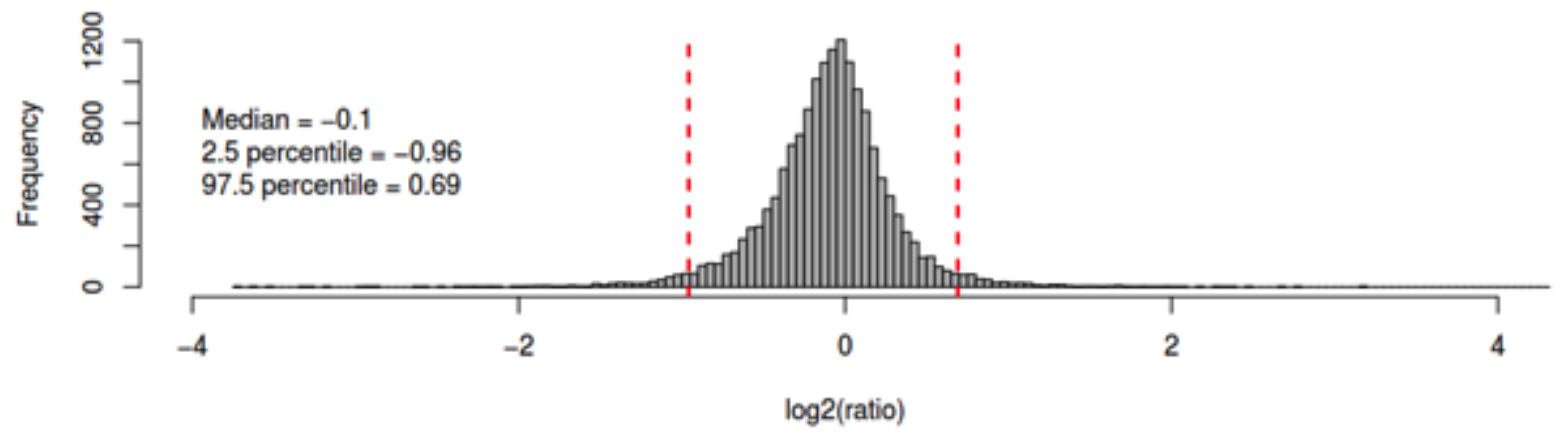

Histogram of all SSB1 QC ratios

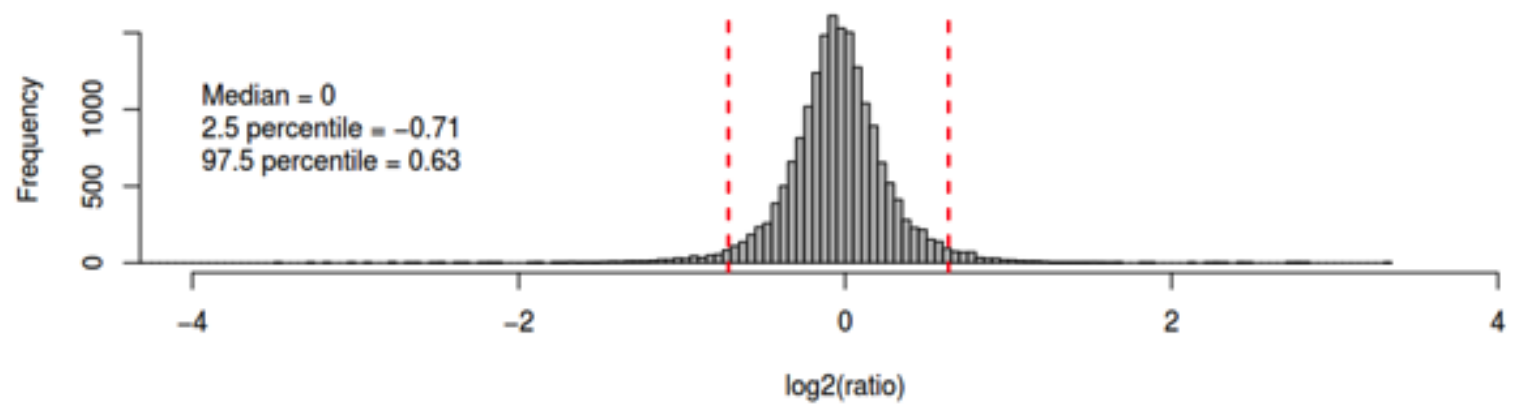

\section{Supplementary Figure 3 Definition of protein fold change thresholds.}

The histograms show the distribution of fold changes between all permutations of the QC sample pairs. The dashed lines show the 2.5 and 97.5 percentile cut-offs that were used for determining the magnitude of a fold change at which a protein was considered to be significantly regulated (in addition to passing the FDRcorrected $p$-value threshold $<0.05$ ). The cut-offs equated to -0.96 (lower bound) and 0.69 (upper bound) for the WT cells, and -0.71 (lower bound) and 0.63 (upper bound) for the mutant cells. 


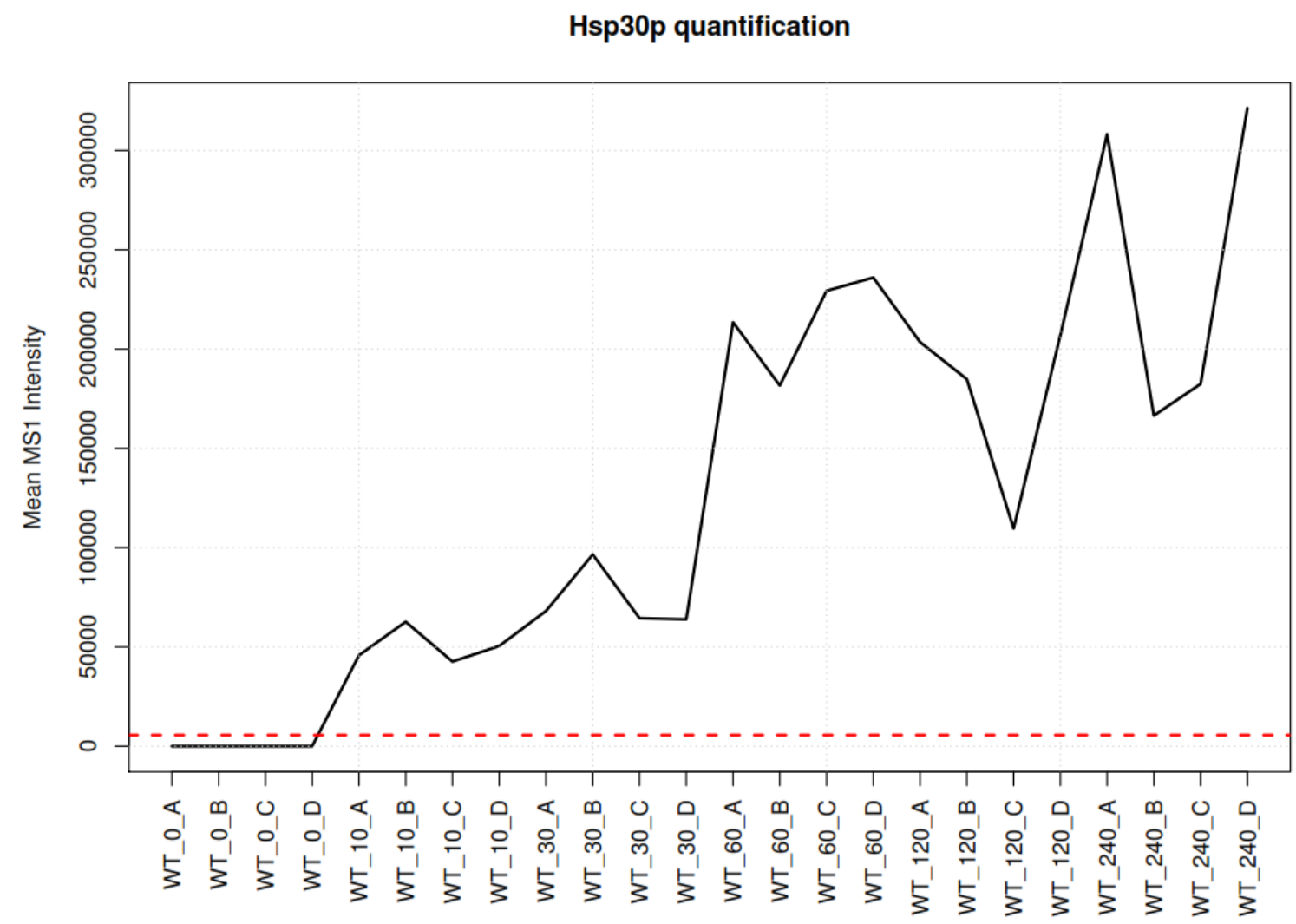

Supplementary Figure 4 Quantification of Hsp30p in the WT samples.

Hsp30p (YCR021C) did not have any detectable peptides in the T0 samples, but was detected and quantified in all later time-points based on three unique peptide sequences: ASGETAIHEPEPEAEQAVEDTA, SITGEVPGIR and LSLTGGFSHHHATDDVEDAAPETK. The solid line represents the average intensity of these three peptides at each time point. The red dashed line represents, for comparison, the intensity value of the lowest quantifiable peptides in this experiment. 


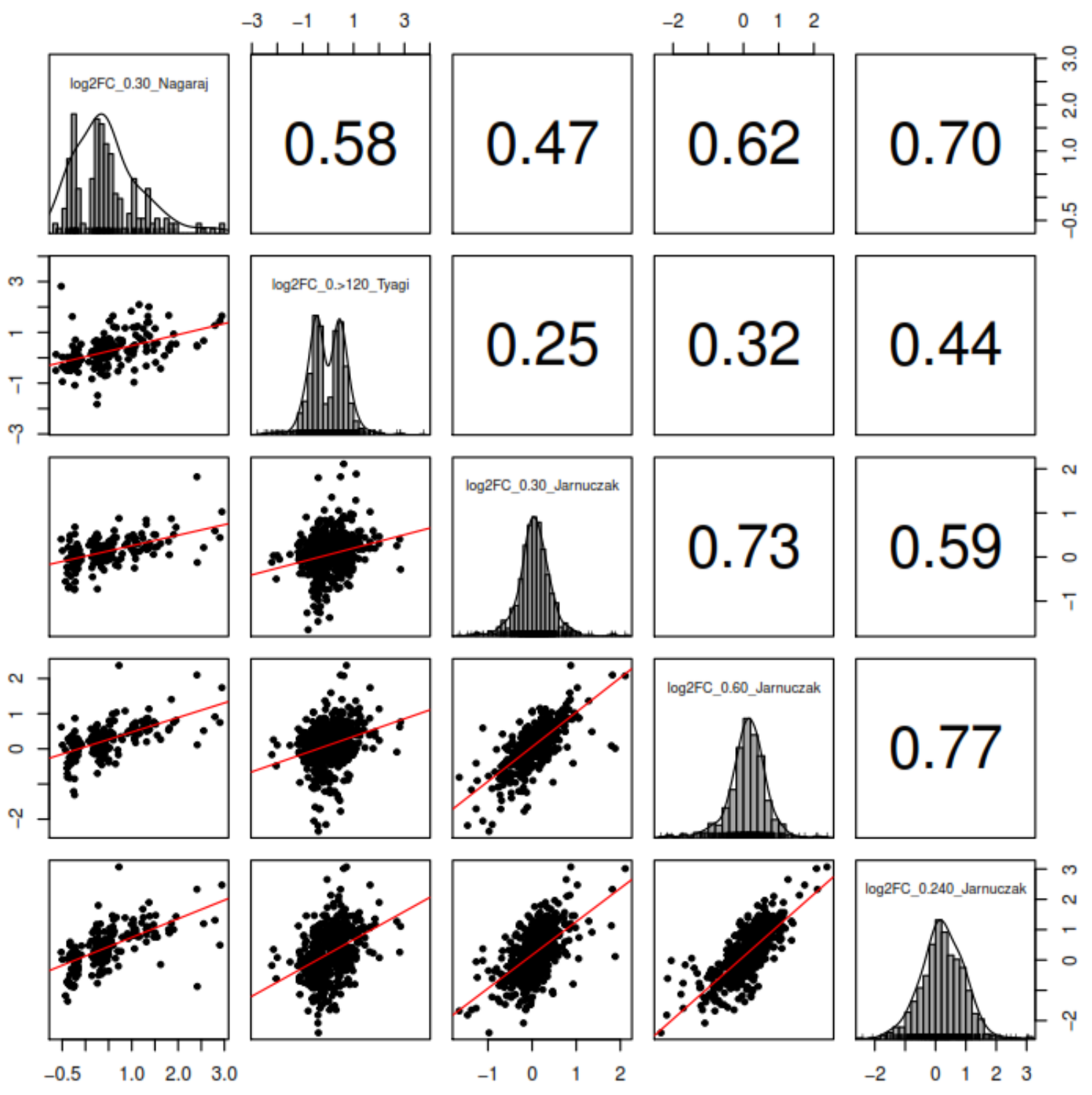

Supplementary Figure 5 Correlation of protein fold changes between this and two other yeast heat shock studies: Nagaraj [12] and Tyagi [13]. 

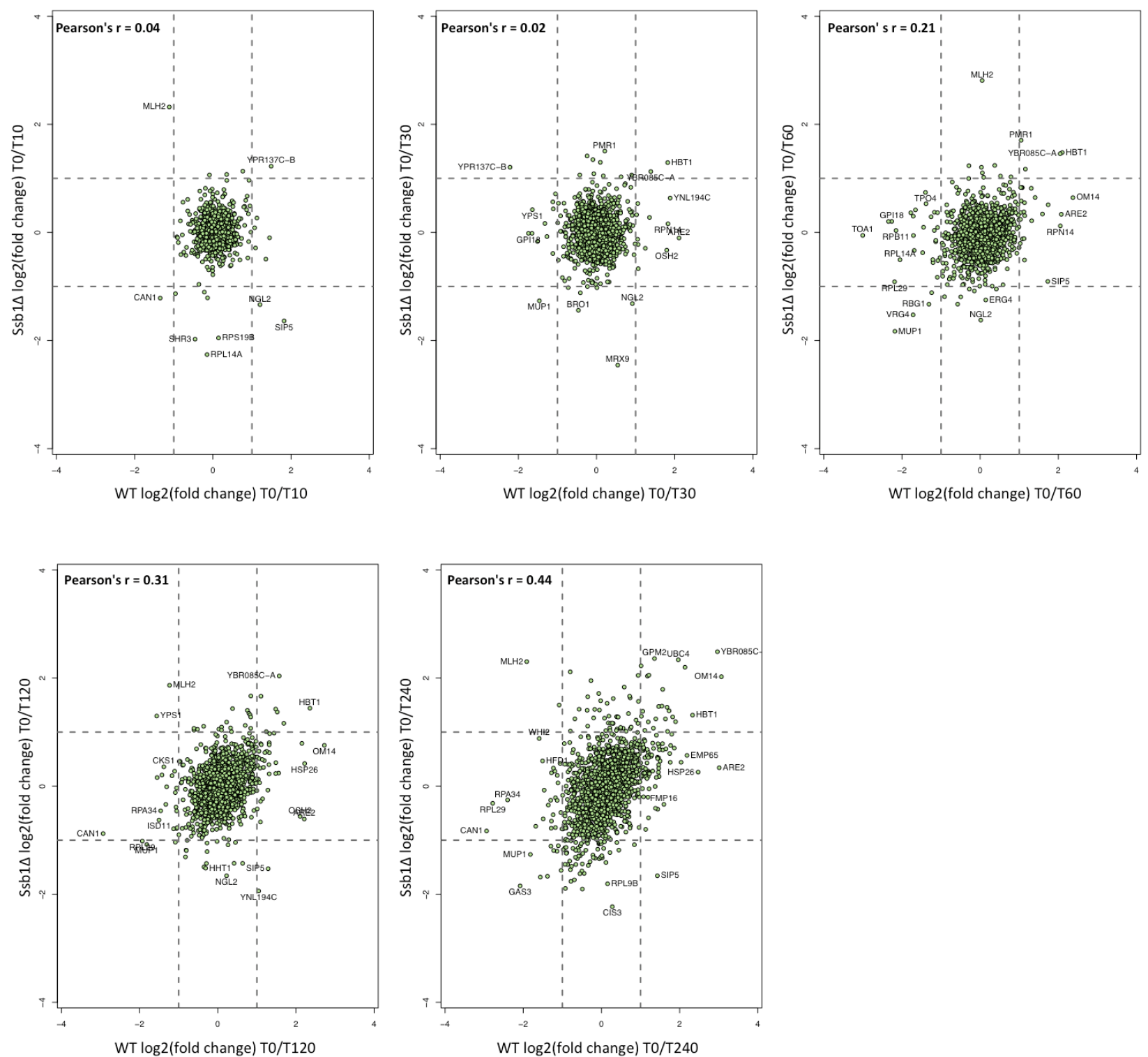

\section{Supplementary Figure 6. Comparison of protein abundance fold changes between wold type and} mutant yeast strains at matched time points after heat shock.

Pairwise comparisons between protein fold changes in the two yeast strains are shown at matched time points after heat shock. Each plot compares the fold change with respect to T0 for each protein in both conditions. The early time points show relatively little remodelling of the proteome has occurred which increases over time post-stress. We note that the correlations between the two strains are modest and effectively non-existant at early time points but increase with time. The data clearly shows a discordance in response between the two strains, whilst some key heat shock response proteins such as Hsp26p remain relatively unaffected in the mutant strain even after 2 or more hours. Interestingly, the novel heat shock responder YBR085C-A is strongly up-regulated in both strains. 


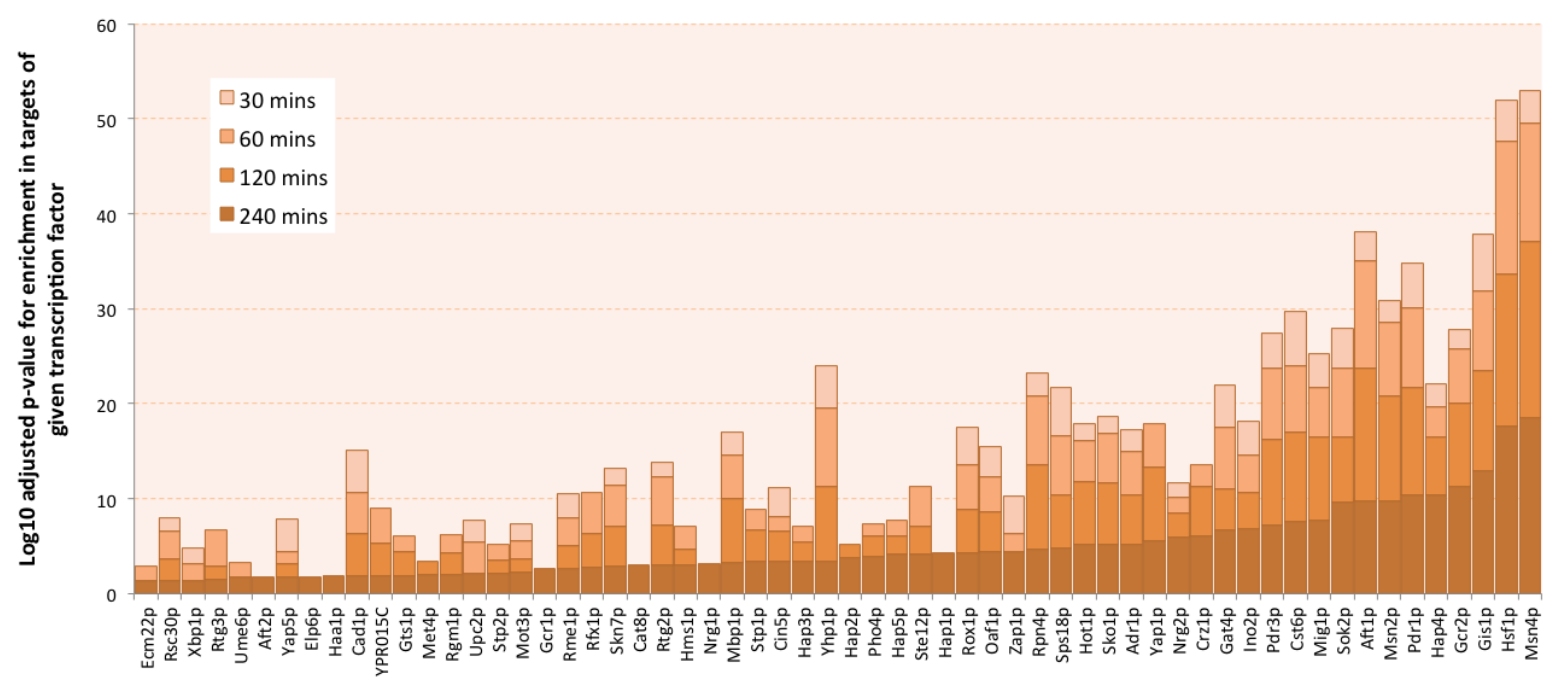

\section{Supplementary Figure 7. Enrichment of transcription factor targets in the upregulated proteome}

\section{over time.}

Enrichment in the up-regulated proteome sets for individual transcription factors was calculated using the GeneCodis [14] website taking the sets of proteins deemed to be up-regulated by MSStats at each time point using an adjusted p-value of $<0.05$ cutoff. The enrichment in these datasets was then estimated by GeneCodis, which uses Yeastract [15] data on known transcription factor-target relationships, against a background total of 1740 proteins observed at least once across all our experiments. Too few proteins were detected with significant increased abundance at 10 mins, and enrichment p-values were therefore only estimated for 30, 60, 120 and 240 minutes after the initial stress. They were then converted to positive integers for convenience (- $\log _{10} p$-value), prior to plotting as a stacked-histogram above, ordered from left to right on increasing significance for the 240 time point. The presence of significant enrichments for targets of many key stress response transcription factors is noted, in particular Hsf1p, Msn2p, Msn4p targets which are well reported heatshock transcription factors. 


\section{REFERENCES}

1. Choi, M.; Chang, C. Y.; Clough, T.; Broudy, D.; Killeen, T.; MacLean, B.; Vitek, O., MSstats: an R package for statistical analysis of quantitative mass spectrometry-based proteomic experiments. Bioinformatics 2014, 30, (17), 2524-6.

2. Harbison, C. T. et al. Transcriptional regulatory code of a eukaryotic genome. Nature 431, 99-104, doi:10.1038/nature02800 (2004).

3. Workman, C. T. et al. A systems approach to mapping DNA damage response pathways. Science 312, 1054-1059, doi:10.1126/science.1122088 (2006).

4. Ni, L. et al. Dynamic and complex transcription factor binding during an inducible response in yeast. Genes \& development 23, 1351-1363, doi:10.1101/gad.1781909 (2009).

5. Borneman, A. R. et al. Transcription factor binding site identification in yeast: a comparison of highdensity oligonucleotide and PCR-based microarray platforms. Functional \& Integrative Genomics 7, 335-345, doi:10.1007/s10142-007-0054-7 (2007).

6. Kasahara, K. et al. Assembly of regulatory factors on rRNA and ribosomal protein genes in Saccharomyces cerevisiae. Molecular and Cellular Biology 27, 6686-6705, doi:10.1128/mcb.0087607 (2007).

7. Capaldi, A. P. et al. Structure and function of a transcriptional network activated by the MAPK Hog1. Nature Genetics 40, 1300-1306, doi:10.1038/ng.235 (2008).

8. Lefrancois, P. et al. Efficient yeast ChIP-Seq using multiplex short-read DNA sequencing. Bmc Genomics 10, 18, doi:10.1186/1471-2164-10-37 (2009).

9. Tan, K. et al. A systems approach to delineate functions of paralogous transcription factors: Role of the Yap family in the DNA damage response. Proceedings of the National Academy of Sciences of the United States of America 105, 2934-2939, doi:10.1073/pnas.0708670105 (2008).

10. Ernst, J., Vainas, O., Harbison, C. T., Simon, I. \& Bar-Joseph, Z. Reconstructing dynamic regulatory maps. Molecular systems biology 3, 13, doi:10.1038/msb4100115 (2007).

11. Schlecht, U. et al. Genome-wide expression profiling, in vivo DNA binding analysis, and probabilistic motif prediction reveal novel Abf1 target genes during fermentation, respiration, and sporulation in yeast. Molecular Biology of the Cell 19, 2193-2207, doi:10.1091/mbc.E07-12-1242 (2008).

12. Nagaraj, N. et al. System-wide Perturbation Analysis with Nearly Complete Coverage of the Yeast Proteome by Single-shot Ultra HPLC Runs on a Bench Top Orbitrap. Mol. Cell. Proteomics 11, 11, doi:10.1074/mcp.M111.013722 (2012).

13. Tyagi, K. \& Pedrioli, P. G. Protein degradation and dynamic tRNA thiolation fine-tune translation at elevated temperatures. Nucleic Acids Res 43, 4701-4712, doi:10.1093/nar/gkv322 (2015).

14. Tabas-Madrid, D.; Nogales-Cadenas, R.; Pascual-Montano, A., GeneCodis3: a non-redundant and modular enrichment analysis tool for functional genomics. Nucleic Acids Res 2012, 40, (Web Server issue), W478-83.

15. Teixeira MC, Monteiro PT, Guerreiro JF, Gonçalves JP, Mira NP, dos Santos SC, Cabrito TR, Palma M, Costa C, Francisco AP, Madeira SC, Oliveira AL, Freitas AT, Sá-Correia I. The YEASTRACT database: an upgraded information system for the analysis of gene and genomic transcription regulation in Saccharomyces cerevisiae. Nucleic Acids Res. 2014 Jan;42(Database issue):D161-6. doi: 10.1093/nar/gkt1015. 